QUARTERLY OF APPLIED MATHEMATICS

VOLUME LXXI, NUMBER 1

MARCH 2013, PAGES 1-36

S 0033-569X(2012)01313-0

Article electronically published on October 15, 2012

\title{
A VARIATIONAL APPROACH TO CARDIAC MOTION ESTIMATION BASED ON COVARIANT DERIVATIVES AND MULTI-SCALE HELMHOLTZ DECOMPOSITION
}

\author{
BY
}

REMCO DUITS (Department of Mathematics/Computer Science and Department of Biomedical Engineering, Eindhoven University of Technology, Den Dolech 2, P.O. Box 513, 5600MB Eindhoven, The Netherlands),

BART JANSSEN (Department of Mathematics/Computer Science, Eindhoven University of Technology, Den Dolech 2, P.O. Box 513, 5600MB Eindhoven, The Netherlands),

ALESSANDRO BECCIU (Department of Biomedical Engineering, Eindhoven University of Technology, Den Dolech 2, P.O. Box 513, 5600MB Eindhoven, The Netherlands),

AND

HANS VAN ASSEN (Department of Biomedical Engineering, Eindhoven University of Technology, Den Dolech 2, P.O. Box 513, 5600MB Eindhoven, The Netherlands)

Abstract. The investigation and quantification of cardiac motion is important for assessment of cardiac abnormalities and treatment effectiveness. Therefore we consider a new method to track cardiac motion from magnetic resonance (MR) tagged images. Tracking is achieved by following the spatial maxima in scale-space of the MR images over time. Reconstruction of the velocity field is then carried out by minimizing an energy functional which is a Sobolev norm expressed in covariant derivatives. These covariant derivatives are used to express prior knowledge about the velocity field in the variational framework employed. Furthermore, we propose a multi-scale Helmholtz decomposition algorithm that combines diffusion and Helmholtz decomposition in one nonsingular analytic kernel operator in order to decompose the optic flow vector field in a divergence-free and a rotation-free part. Finally, we combine both the multi-scale Helmholtz decomposition and our vector field reconstruction (based on covariant derivatives) in a single

Received October 20, 2010.

2010 Mathematics Subject Classification. Primary 55R10, 49M25, 47A05; Secondary 47A10, 49M27.

Key words and phrases. Covariant derivatives, fiber bundles, Helmholtz decomposition, inverse problems, optical flow methods in image analysis, scale space.

Current address: Department of Mathematics/Computer Science and Department of Biomedical Engineering, Eindhoven University of Technology, Den Dolech 2, P.O. Box 513, 5600MB Eindhoven, The Netherlands.

E-mail address: R.Duits@tue.nl

E-mail address: B.J.Janssen@tue.nl

E-mail address: A.Becciu@tue.nl

E-mail address: H.C.v.Assen@tue.nl 
algorithm and show the practical benefit of this approach by an experiment on real cardiac images.

1. Introduction. In cardiology literature [52] it has been noted that deformation of the cardiac wall provides a quantitative indication of the health of the cardiac muscle. Cardiac motion extraction is therefore an important area of research. Monitoring and quantification of irregular cardiac wall deformation may help in early diagnosis of cardiac abnormalities such as ischemia and provides information about the effectiveness of treatment; cf. [10, 46. In order to characterize contraction and dilation of the cardiac muscle, noninvasive acquisition techniques such as magnetic resonance (MR) tagging can be applied. MR tagging allows us to superimpose artificial brightness patterns on the image, which deform according to the cardiac muscle's motion and aid to retrieve motion within the heart walls; cf. Fig. 1. The problem of extracting motion in image sequences
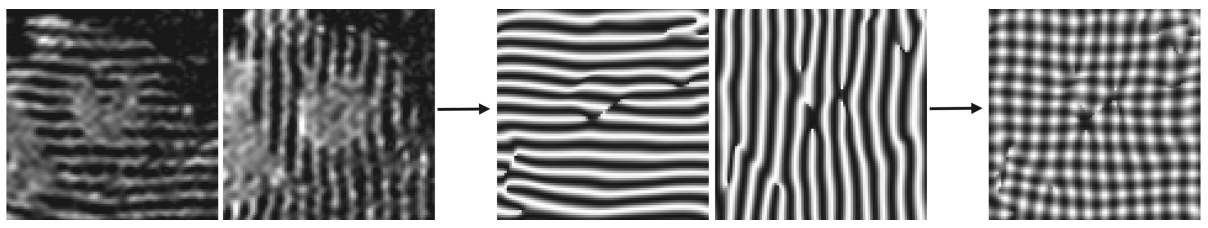

FIG. 1. Left: Short axis view of a patient's left ventricle. Middle: Sine-phase images. Right: Sum of sine-phase images for a fixed time $t$. This sum of sine-phase images serves as input to our algorithms and will be denoted by $f(\mathbf{x}, t)$ where $\mathbf{x}=(x, y) \in \mathbb{R}^{2}, t>0$.

is of primary interest for the computer vision and image analysis community. Optic flow measures apparent motion of moving patterns in image sequences, providing information about spatial displacements of objects in consecutive frames. At the beginning of the eighties, Horn and Schunck introduced a mathematical formulation of optic flow assuming that intensities associated to image objects do not change along the sequence, [29]. This formulation has been referred to as the Optic Flow Constraint Equation (OFCE):

$$
f_{x} u+f_{y} v+f_{t}=0
$$

where $(x, y, t) \rightarrow f(x, y, t): \mathbb{R}^{2} \times \mathbb{R}^{+} \rightarrow \mathbb{R}$ is an image sequence, $f_{x}, f_{y}, f_{t}$ are the spatial and temporal derivatives; $\mathbf{v}(\cdot, t)$ is a vector field on $\mathbb{R}^{2}$ given by $\mathbf{v}(x, y, t)=$ $(u(x, y, t), v(x, y, t))^{T}$, where $u$ and $v$ are unknown and $x, y$ and $t$ are the spatial and temporal coordinates respectively. Since scalar-valued functions $u$ and $v$ are unknown, (1.1) does not have a unique solution, providing the so-called "aperture problem". Horn and Schunck dealt with the aperture problem by proposing a variational framework related to (1.1); cf. [29. For further extensions/improvements, see [6, 19, 55, 59, 63.

The constant brightness assumption (1.1) does not apply to a wide variety of medical imaging problems, such as cardiac motion estimation from MR-tagged images. Furthermore, most of these methods do not take into account physical properties of the velocity field generated by rotation and dilation of the cardiac tissue. Local rotation and contraction of the cardiac muscle can be estimated by Helmholtz decomposition, 
cf. 11, 60, 40, 7, 8, of the vector field. Exploring this decomposition can play a fundamental role in the clinical diagnosis procedure, since it may reveal abnormalities in tissue deformation. Therefore, for applications such as cardiac motion extraction, blood flow calculation and fluid motion analysis, this may lead to more accurate velocity field estimation in comparison to general approaches; cf. [7, 40, 26].

In this work we extract 2-dimensional cardiac wall motion by employing an optic flow method based on features such as spatial extrema in a linear scale space representation of the image sequence. The flow field is reconstructed via a variational method; in the regularization term, we include our multi-scale Helmholtz decomposition and we use covariant derivatives biased by a so-called gauge field. Advantages of this approach are significant:

(i) We do not suffer from the aperture problem.

(ii) Critical points such as spatial maxima are robust with respect to monotonic transformations in the codomain, such as fading, in the image. Therefore, the algorithm can be robustly applied on image sequences (such as tagged MR images) where the intensity constancy is not preserved.

(iii) The proposed technique allows a separate analysis of the divergence-free and rotation-free parts of the cardiac motion by means of a robust multi-scale Helmholtz decomposition.

(iv) The algorithm has the advantages provided by a multi-scale approach:

- A scale selection scheme for the feature points (extrema) is included, where we take into account both topological transitions and spatial dislocation of extremal paths in scale space. For details, see [12, ch:4.1].

- We avoid typical grid artifacts by analytical precomputation of the concatenation of linear diffusion and Helmholtz decomposition; cf. Cuzol et al. [8]. Besides the gradient of the potential in [8] we also express the subsequent derivative operators in Gaussian derivatives. This yields a single analytic vector-valued kernel that can be precomputed (avoiding vortex-particle approximations). We do not use discrete multi-scale Helmholtz decompositions, cf. [56, which act by means of nonlinear diffusions on the potentials, since we need to keep track of the scale by means of linear diffusion on the field.

(v) We obtain a better flow field reconstruction, compared to similar techniques cf. [5, 34, by replacing standard derivatives by covariant derivatives, which allows us to incorporate prior knowledge (based on features) in the regularization term. An alternative approach to include prior knowledge in the regularization term can be found in the work by Nir et al. 48.

(vi) Both the computation of the gauge field and the reconstruction framework are stable linear operators (due to the coercivity of the covariant Laplacian).

Throughout this article we will consider cardiac motion. Our general technique can also be applied to cardiac strain and deformation, which is also relevant in cardiac imaging; cf. [58, 20]. However, strain can also be directly computed from frequency fields using (enhanced) Gabor transforms; cf. [13]. 
Outline of algorithm and article. An overview of the proposed algorithm is provided in Figure 2 and every step is described as follows. Section 3 describes the methodology used to calculate velocity features including a scale selection. Section 4 is dedicated to the multi-scale Helmholtz decomposition of vector fields, where we analytically compute the effective kernel operator that arises from the concatenation of the (commuting) linear diffusion operator and the Helmholtz decomposition operator.

In Sections 5. 6] we introduce the concept of covariant derivatives. Subsequently, in Section 7 we investigate the covariant Laplacian and in Section 8 we derive its associated Green's function of the covariant Laplacian. Furthermore, we deduce a lower bound on the spectrum of the covariant Laplacian.

In Section 9 we consider motion field reconstruction by minimization of an energy functional where the data term is obtained by our approach explained in Section 3 and where the smoothness term is expressed in the covariant derivatives of Section 66 The motion field reconstruction is obtained by solving continuous Euler-Lagrange equations. In Section 10 we also derive the corresponding discrete equations arising by expansion in a B-spline basis. Stability is manifest, both in the continuous and discrete Euler-Lagrange systems as we show in Section 11 where we rely on our results in Section 8 .

Then in Section 12 we put everything together and include the multi-scale Helmholtz decomposition in the motion field reconstruction. Here we distinguish between two options, a pragmatic one which consists of two separate reconstruction algorithms for the divergence-free and rotation-free parts and a more elaborate approach where we merge everything into a single energy minimization yielding a related, but more difficult, EulerLagrange system as explained in Section 14

Finally in Section 15 we apply our algorithm to real data obtained from a patient and a healthy volunteer. For further details on experiments on phantoms with known ground truth and extensive assessment of the algorithm performance (qualitatively and quantitatively) we refer to our technical report [12. These experiments clearly show the advantage of including both covariant derivatives and Helmholtz decomposition. In this paper we rather put emphasis on the mathematical underpinning, stability and fundamental properties of our approach.

2. Input: Tagged MR images. Tagging is a noninvasive technique based on locally perturbing the magnetization of the cardiac tissue via radio frequency pulses. MR tags are artificial patterns, which appear as dark stripes on the MR images with the aim to improve the visualization of the deforming tissue 62. An example of a tagged heart image is displayed in Figure 1, column 1. In order to increase the number of tags in the image, Axel and Dougherty [2] spatially modulated the degree of magnetization in the cardiac tissue, whereas Osman et al. 49. proposed the so-called harmonic phase (HARP) method, which converts MR images into phase images. In our experiments we apply a similar technique where we extract sine-phase images by means of Gabor filters [22, 59] (Figure 1, column 2). Such images allow us to extract feature points such as maxima, minima and saddles with high accuracy. The calculated sine-phase images have been combined in order to create a chessboard-like pattern. Throughout this article we will apply our methods to phase images as depicted in Figure 1 column 3. 


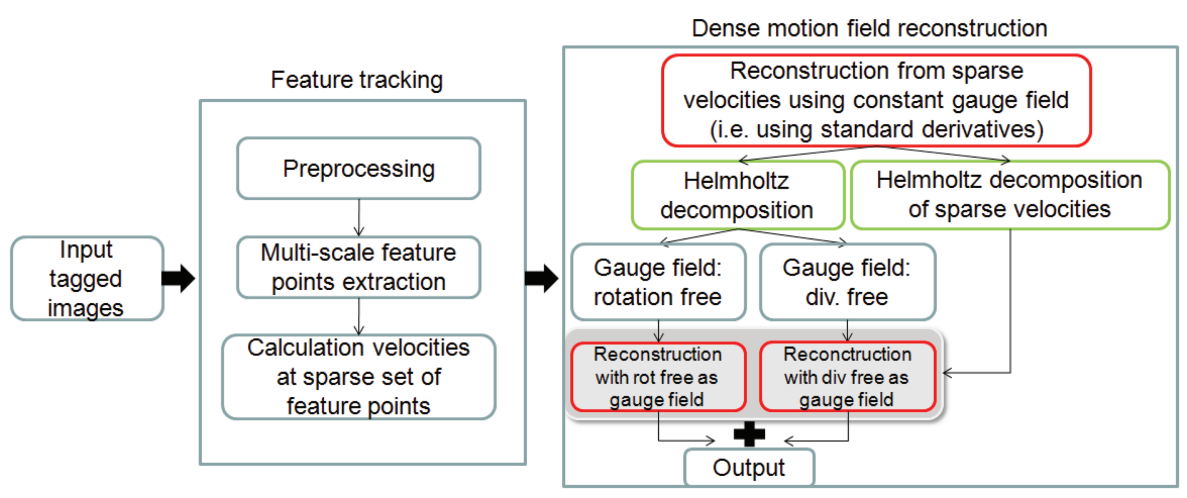

Fig. 2. Overview of the algorithm. Input tagged images and first preprocessing steps are discussed in Section 2 The feature tracking procedure is described in Section 3 Sections 5 and 6 explain the concept of covariant derivatives, and in Section 4 we present our multi-scale Helmholtz decomposition algorithm. The box on the right shows how these two techniques are applied in the dense motion field reconstruction that we present in Sections 9 10 and 12

3. Computation of velocity features via critical paths in scale space. The so-called Gaussian scale space representation $I: \mathbb{R}^{2} \times \mathbb{R}^{+} \rightarrow \mathbb{R}$ of a $2 \mathrm{D}$ static image $\mathbf{x} \mapsto f(\mathbf{x}) \in \mathbb{L}_{2}\left(\mathbb{R}^{2}\right)$ is defined by the spatial convolution of the image with a Gaussian kernel

$$
I(\mathbf{x}, s)=\left(f * \phi_{s}\right)(\mathbf{x}), \text { with } \phi_{s}(\mathbf{x})=\frac{1}{4 \pi s} \exp \left(-\frac{\|\mathbf{x}\|^{2}}{4 s}\right), s>0,
$$

where $\mathbf{x}=(x, y) \in \mathbb{R}^{2}$ and where $s>0$ represents the scale of observation [30, 61, 42, 43, 39, 54, 16. Note that (3.1) is the solution of a diffusion system on the upper half-space $s>0$, so $\frac{\partial}{\partial s} I=\Delta I$ and $\lim _{s \downarrow 0} I(\cdot, s)=f, \lim _{s \rightarrow \infty} I(\cdot, s)=0$ where both limits are taken in the $\mathbb{L}_{2}$-sense. This procedure naturally extends to a multiple scale representation of a dynamic image $(x, y, t) \mapsto f(x, y, t)$ :

$$
I(x, y, s, t):=\left(\phi_{s} * f(\cdot, \cdot, t)\right)(x, y), \quad t, s>0, \mathbf{x}=(x, y) \in \mathbb{R}^{2} .
$$

For each fixed time $t>0$ we compute the critical paths in $\mathbb{R}^{2} \times \mathbb{R}^{+}$, where the spatial gradient vanishes $\nabla I(\mathbf{x}, s, t)=\mathbf{0}$. We used a sub-pixel accurate method to determine the critical paths based on topological numbers; for details, see [53, 12. By means of Morse singularity theory 9, 18, one can deduce that these critical paths generically vanish 1 at so-called top-points as the scale increases. These top-points are given by

$$
\nabla I(\mathbf{x}, s, t)=0 \text { and } \operatorname{det} H I(\mathbf{x}, s, t)=I_{x x}(\mathbf{x}, s, t) I_{y y}(\mathbf{x}, s, t)-\left(I_{x y}(\mathbf{x}, s, t)\right)^{2}=0 .
$$

In the visualization of critical paths in scale space, the scale $s$ is parameterized logarithmically as

$$
s / s_{\min }=e^{2 \tau} \Leftrightarrow \tau=\frac{1}{2} \log \left(s / s_{\min }\right)
$$

\footnotetext{
${ }^{1}$ During diffusion, creation of critical paths occurs as well, cf. 9, 18, but these topological transitions are less frequent and are usually followed by an annihilation at a slightly higher scale.
} 
see Figure 3. Inspired by the general works on image reconstruction [47, 41, 38, we have shown [35, 14, 11, 31, 32] that up to second order, differential structure at these points allows a visually appealing reconstruction of the image at scale $s=0$ (via orthogonal projection). This suggests that top-points are visually descriptive feature points.

On the one hand, we need large scales in our feature selection for robust velocity estimation. See Figure 3 and Figure 4. On the other hand, we need to avoid extreme dislocation of spatial extrema within scale space and instable parts of critical curves in our feature selection; cf. Figure 5 .
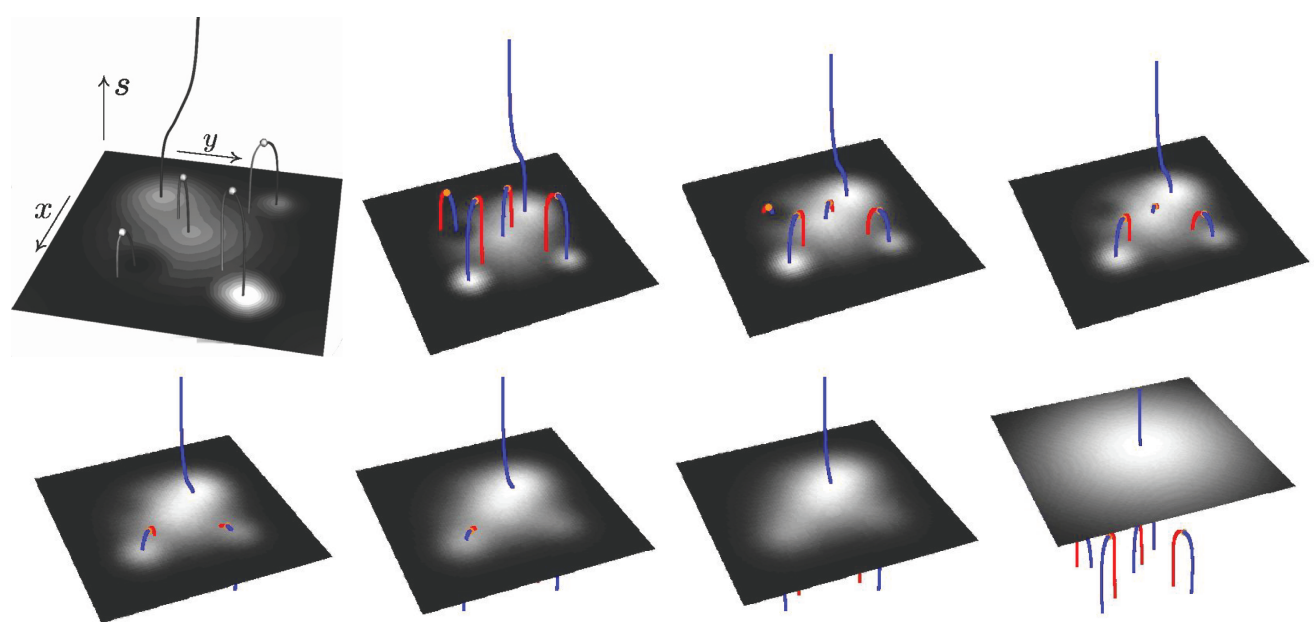

FIG. 3. Topological transitions at so-called top-points, cf. Eq. (3.2), in scale space representations of images. In this case one observes only annihilations where a spatial saddle-branch (red) merges with a spatial extremal branch (blue).

Typically, the slope of the tangent vector along the $q$ th connected critical branch $s \mapsto\left(\mathbf{x}_{s}^{q}(t), s\right)$ in scale space provides a measure on the stability and dislocation. Here $q=1, \ldots, N_{B}$ indexes the branch of a certain critical point. At scale 0 an extremal branch of a strong extremum (i.e. $s_{q}^{*} \gg 0$ ) is nearly vertical whereas at top-point scale $s_{q}^{*}$ the slope is horizontal; cf. [18].

Therefore, for each fixed time $t>0$, we propose the following scale selection per $q$-th critical branch:

$$
\begin{aligned}
s_{q}(t):=\max & \left\{s=s_{\min } e^{2 \tau} \in\left[0, s_{q}^{*}\right) \mid \text { for all } s^{\prime}=s_{\min } e^{2 \tau^{\prime}} \in[0, s)\right. \text { we have } \\
& \left.\arccos \left(\frac{\beta}{\sqrt{\left\|\left.\frac{d}{d \tau} \mathbf{x}_{s}^{q}(t)\right|_{\tau=\tau^{\prime}}\right\|^{2}+\beta^{2}}}\right)<\vartheta\right\},
\end{aligned}
$$

where the tangent vector along the critical curve in scale space is given by

$$
\frac{d}{d \tau} \mathbf{x}_{s}^{q}(t)=2 s \frac{d}{d s} \mathbf{x}_{s}^{q}(t)=-2 s\left[H I\left(\mathbf{x}_{s}^{q}(t), s, t\right)\right]^{-1} \Delta \nabla I\left(\mathbf{x}_{s}^{q}(t), s, t\right),
$$

as derived in [43, p.189], where $\vartheta$ is an a priori threshold angle, and where $\beta$ is a parameter with physical dimension [length] according to the dimensionally consistent, translation 


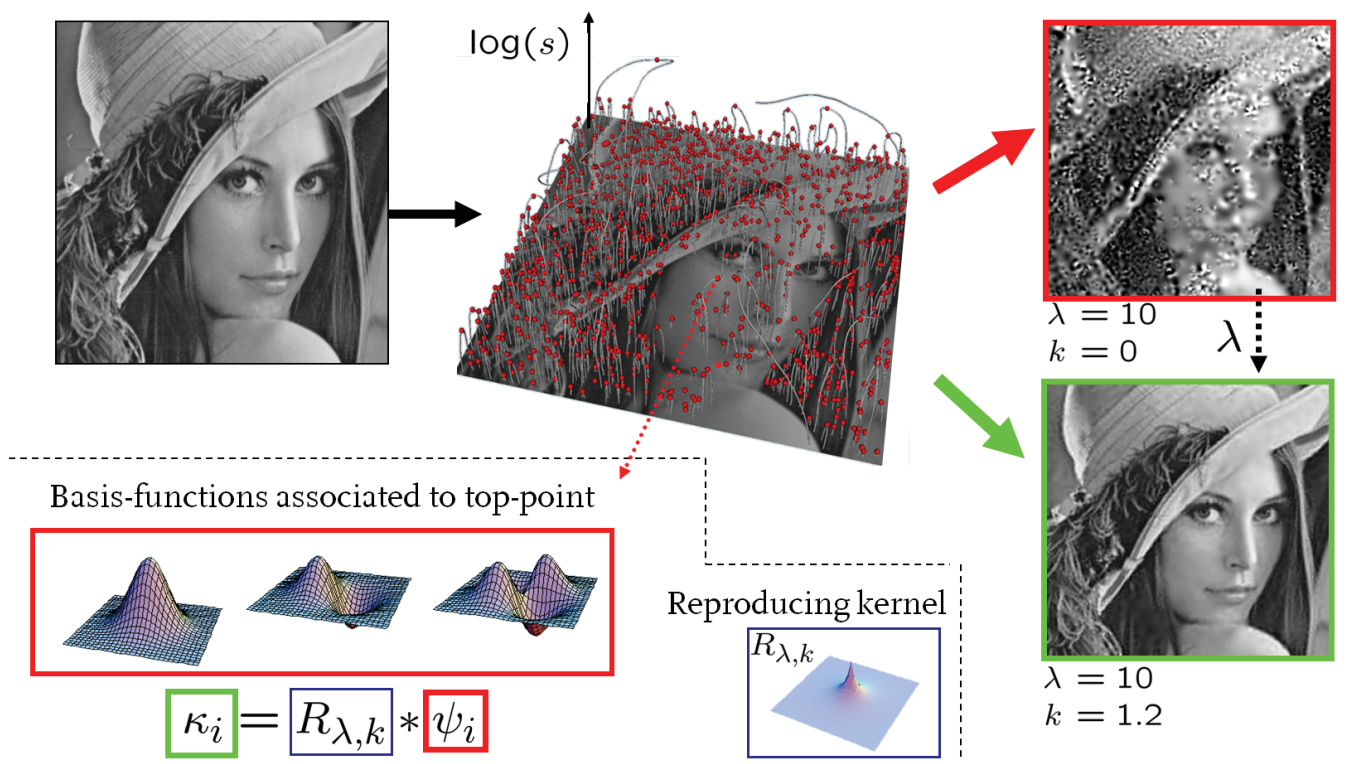

FIG. 4. Reconstruction from top-points of static images in a nutshell: The up to $n$ th-order derivatives at each top-point provide a set of continuous linear functionals $\left\{f \mapsto\left(\psi_{i}, f\right)\right\}_{i=1}^{N}$ on the original image. Their Sobolev inner product Riesz representatives are obtained from the $\mathbb{L}_{2}$ Riesz representative by convolution with the reproducing kernel $R_{\lambda, k}$ of the Sobolev space endowed with the inner product $\left(g_{1}, g_{2}\right)_{k, \lambda}=\left(g_{1}, g_{2}\right)+\lambda\left(\Delta^{k / 2} g_{1}, \Delta^{k / 2} g_{2}\right)$, where $k>1$ denotes the order and $\lambda$ is a regularization parameter. The minimizer of $g \mapsto(g, g)_{k, \lambda}$ coincides with orthogonal projection of the input image on the span of the corresponding Riesz representatives $\left\{\kappa_{i}\right\}_{i=1}^{N}$. Visually most appealing results are obtained for $1<k<1.5, \lambda \approx 10$ for bounded domain Sobolev norms, 32, 31, while including other features [41, 47. such as the top-points of the Laplacian [36 31. Using covariant derivatives further improves the result in our faster coarse to fine iterative scheme; cf. 33, 31.

and scaling invariant metric tensor

$$
\mathrm{d} x \otimes \mathrm{d} x+\mathrm{d} y \otimes \mathrm{d} y+\beta^{2} \mathrm{~d} \tau \otimes \mathrm{d} \tau=\mathrm{d} x \otimes \mathrm{d} x+\mathrm{d} y \otimes \mathrm{d} y+\beta^{2}(2 s)^{-2} \mathrm{~d} s \otimes \mathrm{d} s
$$

that we impose on scale space $\mathbb{R}^{2} \times \mathbb{R}^{+}$to introduce slope in scale space. In our experiments we set $\beta=(\Delta \tau)^{-1} \sqrt{(\Delta x)^{2}+(\Delta y)^{2}}$, where $\Delta x, \Delta y, \Delta \tau$ denote step sizes.

In tracking critical points over time we use the fact that those points satisfy

$$
\nabla I\left(\mathbf{x}_{s}^{q}\left(t_{k}\right), s, t_{k}\right)=0
$$

where $\nabla$ denotes the spatial gradient and $I\left(\mathbf{x}_{s}^{q}, s, t_{k}\right)$ represents intensity at position $\mathbf{x}_{s}^{q}$, scale $s$ and time frame $t_{k}=k \Delta t$, where $\mathbf{x}_{s}^{q}(t)=\mathbf{x}_{s}^{q}(0)+\int_{0}^{t} \widetilde{\mathbf{v}}_{q}\left(\mathbf{x}_{s}^{q}(\tau)\right) d \tau$ such that $\frac{d}{d t} \mathbf{x}_{s}^{q}(t)=\widetilde{\mathbf{v}}_{q}\left(\mathbf{x}_{s}^{q}(t)\right)$ with $\widetilde{\mathbf{v}}(\mathbf{x}(t))=\mathbf{v}(\mathbf{x}(t), t)$. Index $k=1, \ldots, K$ corresponds to the time frame number. The amount of frames and critical points are denoted by respectively 

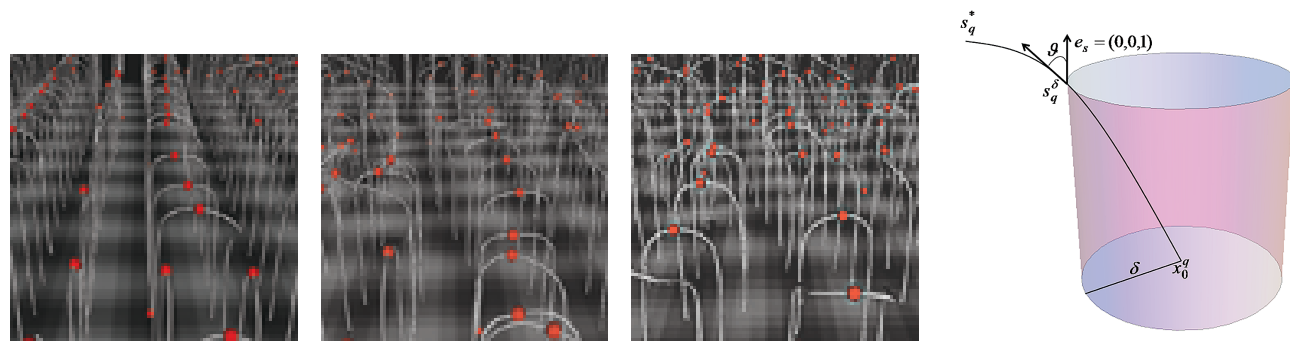

FIG. 5. Left: Three subsequent frames of the heart chessboard representation illustrated together with the respective critical paths (white lines) and top-points (red dots), where we used the software ScaleSpaceVis [37]. Due to the symmetry of the chessboard pattern, critical branches annihilate with different neighbors through the sequence and top-points show strong displacements. Right: Therefore we apply a scale selection per critical branch, see (3.3), which avoids ill-posed spatial dislocations.

$K$ and $N_{B}$. The critical point velocities [43, 18] are given by:

$$
\left[\begin{array}{l}
\widetilde{u}\left(\mathbf{x}_{s}^{q}\left(t_{k}\right)\right) \\
\widetilde{v}\left(\mathbf{x}_{s}^{q}\left(t_{k}\right)\right)
\end{array}\right]=\left[\begin{array}{l}
u\left(\mathbf{x}_{s}^{q}\left(t_{k}\right), t_{k}\right) \\
v\left(\mathbf{x}_{s}^{q}\left(t_{k}\right), t_{k}\right)
\end{array}\right]=-\left(H I\left(\cdot, \cdot, t_{k}\right)\left(\mathbf{x}_{s}^{q}, s\right)\right)^{-1} \frac{\partial\left(\nabla I\left(\mathbf{x}_{s}^{q}, s, t_{k}\right)\right)^{T}}{\partial t_{k}},
$$

where $H I$ denotes the spatial Hessian matrix of image $I$. The vector $\left(\widetilde{u}\left(\mathbf{x}_{s}^{q}\left(t_{k}\right)\right), \widetilde{v}\left(\mathbf{x}_{s}^{q}\left(t_{k}\right)\right)\right)$ denotes the critical point velocity vector at position $\mathbf{x}_{s}^{q}\left(t_{k}\right)$ at the time $t_{k}$ at scale $s>0$.

In the remainder of this article we will abbreviate these critical point velocities by

$$
\mathbf{d}_{q}^{k}:=\left(\begin{array}{c}
d_{q}^{k, 1} \\
d_{q}^{k, 2}
\end{array}\right):=\left(\begin{array}{c}
\widetilde{u}\left(\mathbf{x}_{s=s_{q}\left(t_{k}\right)}^{q}\left(t_{k}\right)\right) \\
\widetilde{v}\left(\mathbf{x}_{s=s_{q}\left(t_{k}\right)}^{q}\left(t_{k}\right)\right)
\end{array}\right),
$$

where we combine the tracking over time, (3.5) and the scale selection, (3.3).

4. Vector field decomposition. The behavior of cardiac muscle is characterized by twistings and contractions, which can be studied independently by an application of the well-known Helmholtz decomposition, 60. Given a bounded domain $\Omega \subseteq \mathbb{R}^{3}$ and a smooth vector field $\mathbf{v}$, in our case $\mathbf{v}=\mathbf{v}(\cdot, t)$, the reconstructed cardiac motion field at a fixed time $t>0, \mathbf{v} \in \mathbf{C}^{0}(\bar{\Omega})$ and $\mathbf{v} \in \mathbf{C}^{1}(\Omega)$, where $\bar{\Omega}=\Omega \cup \partial \Omega$, there exist functions $\Phi \in C^{1}(\Omega)$ and $\mathbf{A} \in \mathbf{C}^{1}(\bar{\Omega})$ such that

$$
\mathbf{v}(\mathbf{x})=\nabla \Phi(\mathbf{x})+\nabla \times \mathbf{A}(\mathbf{x})
$$

and $\nabla \cdot \mathbf{A}(\mathbf{x})=0$, where $\mathbf{x}=(x, y, z) \in \mathbb{R}^{3}$. The functions $\Phi$ and $\mathbf{A}$ are the so-called scalar potential and vector potential of $\mathbf{v}$. However, in our cardiac MR tagging application we consider $\Omega \subseteq \mathbb{R}^{2}$ and in $\mathbb{R}^{2}$ one does not have an outer product at hand and therefore we need the following definition and remarks.

Definition 4.1. Recall that the rotation of a vector field in 3D is, in Euclidean coordinates, expressed as

$$
\operatorname{rot} \mathbf{v}=\nabla \times \mathbf{v}=\left(\begin{array}{c}
\partial_{y} v^{3}-\partial_{z} v^{2} \\
\partial_{z} v^{1}-\partial_{x} v^{3} \\
\partial_{x} v^{2}-\partial_{y} v^{1}
\end{array}\right)
$$


where we use the short notation $\partial_{x^{i}}:=\frac{\partial}{\partial x^{i}}$, for $x^{1}=x, x^{2}=y, x^{3}=z$, for partial derivatives. We define the rotation of a $2 \mathrm{D}$-vector vector field in Euclidean coordinates as follows:

$$
\operatorname{rot} \mathbf{v}:=\partial_{x} v^{2}-\partial_{y} v^{1}
$$

and we define the rotation of a scalar field in Euclidean coordinates 2 by

$$
\widetilde{\operatorname{rot}} F:=\left(\begin{array}{l}
\partial_{y} F \\
-\partial_{x} F
\end{array}\right) \text {. }
$$

Helmholtz decomposition in 3D is adapted to 2D by replacing the rotation (4.2) consistently by respectively (4.3) and (4.4). For example, the identity underlying 3D-Helmholtz decomposition is $\mathbf{v}=\Delta \boldsymbol{\xi}=\operatorname{grad} \operatorname{div} \boldsymbol{\xi}-\operatorname{rot} \operatorname{rot} \boldsymbol{\xi}$ which now in $2 \mathrm{D}$ becomes

$$
\mathbf{v}=\Delta \boldsymbol{\xi}=\operatorname{grad} \operatorname{div} \boldsymbol{\xi}-\widetilde{\operatorname{rot}} \operatorname{rot} \boldsymbol{\xi}=\operatorname{grad} \Phi+\widetilde{\operatorname{rot}} A \text {. }
$$

Lemma 4.2. A particular solution $(\Phi, A)$ of $\mathbf{v}=\operatorname{grad} \Phi+\widetilde{\operatorname{rot}} A$ is given by

$$
\begin{aligned}
& \Phi=\operatorname{div} \boldsymbol{\xi} \text { and } A=-\operatorname{rot} \boldsymbol{\xi}, \text { with } \\
& \boldsymbol{\xi}(\mathbf{x})=\left(\left(G^{2 D} *\left(1_{\Omega} v^{1}\right)\right)(\mathbf{x}),\left(G^{2 D} *\left(1_{\Omega} v^{2}\right)\right)(\mathbf{x})\right)=\int_{\Omega} G^{2 D}\left(\mathbf{x}-\mathbf{x}^{\prime}\right) \mathbf{v}\left(\mathbf{x}^{\prime}\right) \mathrm{d} \mathbf{x}^{\prime},
\end{aligned}
$$

where $1_{\Omega}$ denotes the indicator function on $\Omega$ and where the fundamental solution for the 2-dimensional Laplacian is given by

$$
G^{2 D}\left(\mathbf{x}-\mathbf{x}^{\prime}\right)=\frac{1}{2 \pi} \ln \left\|\mathbf{x}-\mathbf{x}^{\prime}\right\| .
$$

Proof. Note that $\Delta \boldsymbol{\xi}=\mathbf{v}$ from which the result follows by (4.5).

The decomposition (4.6) is not unique; e.g., one can replace $\boldsymbol{\xi} \mapsto \boldsymbol{\xi}+\mathbf{h}$ where $\mathbf{h}$ is some arbitrary harmonic vector field. Furthermore, if both the divergence and rotation of a vector field vanish, then this vector field equals the gradient of some harmonic function. However, the decomposition is unique if we prescribe the field to vanish at the boundary and if moreover we prescribe both the divergence-free and rotation-free parts at the boundary; for details, see [12, Lemma 1]. In practice we cannot assume that the field vanishes at the boundary. Therefore we subtract the harmonic infilling and write

$$
\begin{gathered}
\mathbf{v}(\mathbf{x})=\nabla \int_{\Omega} \nabla \cdot G^{2 D}\left(\mathbf{x}-\mathbf{x}^{\prime}\right) \widetilde{\mathbf{v}}\left(\mathbf{x}^{\prime}\right) \mathrm{d} \mathbf{x}^{\prime} \\
-\widetilde{\operatorname{rot}} \int_{\Omega} \operatorname{rot} G^{2 D}\left(\mathbf{x}-\mathbf{x}^{\prime}\right) \widetilde{\mathbf{v}}\left(\mathbf{x}^{\prime}\right) \mathrm{d} \mathbf{x}^{\prime}+\boldsymbol{\psi}(\mathbf{x}),
\end{gathered}
$$

where the vector field $\widetilde{\mathbf{v}}(\mathbf{x})=\mathbf{v}(\mathbf{x})-\boldsymbol{\psi}(\mathbf{x})$ vanishes at the boundaries, with $\boldsymbol{\psi}=\left(\left.\mathbf{v}\right|_{\partial \Omega}\right)_{\mathcal{H}}$ the unique harmonic infilling (as defined below).

Definition 4.3. The harmonic infilling $\boldsymbol{\psi}=\left(\left.\mathbf{v}\right|_{\partial \Omega}\right)_{\mathcal{H}}$ of the field $\left.\mathbf{v}\right|_{\partial \Omega}$ restricted to the boundary $\partial \Omega$ is the unique solution to

$$
\left\{\begin{array}{l}
\triangle \boldsymbol{\psi}(\mathbf{x})=0, \quad \mathbf{x} \in \Omega \\
\left.\boldsymbol{\psi}\right|_{\partial \Omega}=\left.\mathbf{v}\right|_{\partial \Omega}
\end{array}\right.
$$

\footnotetext{
${ }^{2}$ Recall that the coordinate-free definition of the rotation operator in $\mathbb{R}^{3}$ is given by $\mathcal{G}^{-1} * d \mathcal{G}$, where $\mathcal{G}: T\left(\mathbb{R}^{n}\right) \mapsto\left(T\left(\mathbb{R}^{n}\right)\right)^{*}$ is the linear operator given by $\mathcal{G}\left(\partial_{x^{i}}\right)=\mathrm{d} x^{i}$ and where $*$ denotes the Hodge star operator and where $d$ denotes the exterior derivative. Similarly on $\mathbb{R}^{2}$ the operator given by Eq. (4.3) can be written as $* d \mathcal{G}$ and the operator given by Eq. (4.4) can be written as $\mathcal{G}^{-1} * d$.
} 
As the Helmholtz decomposition (4.6) is not unique, we briefly motivate our particular choice of decomposition (4.8) by the subsequent remark.

REMARK 4.4. A different choice to determine $\boldsymbol{\xi}$ uniquely is to impose $\left.\boldsymbol{\xi}\right|_{\partial \Omega}=0$ besides $\Delta \boldsymbol{\xi}=\mathbf{v}$. This would boil down to

$$
\tilde{\mathbf{v}}=\operatorname{grad} \operatorname{div} \mathcal{D} \tilde{\mathbf{v}}-\widetilde{\operatorname{rot}} \operatorname{rot} \mathcal{D} \tilde{\mathbf{v}}
$$

where $\mathcal{D}$ is the Dirichlet operator, i.e., $\boldsymbol{\xi}=\mathcal{D} \tilde{\mathbf{v}} \Leftrightarrow \Delta \boldsymbol{\xi}=\tilde{\mathbf{v}}$ and $\left.\boldsymbol{\xi}\right|_{\partial \Omega}=\mathbf{0}$. However, the Dirichlet kernel on a rectangle, see [32, App. $\tilde{\mathrm{A}}$ ], is not as tangible as the convolution operator $\boldsymbol{\xi}=\mathcal{G} \tilde{\mathbf{v}}=\left(G^{2 D} * \tilde{v}_{1} 1_{\Omega}, G^{2 D} * \tilde{v}_{2} 1_{\Omega}\right)$ with $\tilde{\mathbf{v}}=\left(\tilde{v}_{1}, \tilde{v}_{2}\right)$ with kernel $G^{2 D}(\mathbf{x}-\mathbf{y})$. Note that $\Delta \mathcal{D}=\Delta \mathcal{G}=I$ and akin to (4.9) we can rewrite (4.8) as

$$
\tilde{\mathbf{v}}=\operatorname{grad} \operatorname{div} \mathcal{G} \tilde{\mathbf{v}}-\widetilde{\operatorname{rot}} \operatorname{rot} \mathcal{G} \tilde{\mathbf{v}} \text {. }
$$

Besides, operator $\mathcal{D}$ is (in contrast to operator $\mathcal{G}$ ) not translation covariant.

4.1. Multi-scale Helmholtz decomposition of the optical flow field. Instead of using standard derivatives in the Helmholtz decomposition (4.8) and (4.10), we can differentiate the involved Green's function by Gaussian derivatives, i.e. by convolving with a derivative of a Gaussian kernel. In this procedure the kernel is affected by a diffusion, which depends on a parameter $s=\frac{1}{2} \sigma^{2}$, the scale. This diffusion removes the singularity at the origin and, therefore, discretization artifacts. Furthermore, the remaining differential operators grad and $\widetilde{\text { rot }}$ can be expressed in Gaussian derivatives as well.

By means of the theorem below one can combine everything in a single analytic kernel operator. This yields a much more accurate method for multi-scale Helmholtz decomposition than standard numerical discretizations of (4.8) and (4.10). For experiments and comparison between different numerical computations of the Helmholtz decomposition by means of an analytic ground truth example, we refer to our technical report [12, ch.5.2], where our approach (4.11) clearly outperforms the other approaches in terms of angular errors and relative $\ell_{\infty}$-errors.

TheOREm 4.5. Given a vector field $\mathbf{v}$ on $\Omega \subset \mathbb{R}^{2}$ we set $\tilde{\mathbf{v}}(\mathbf{x}):=\left(\mathbf{v}(\mathbf{x})-\left(\left.\mathbf{v}\right|_{\partial \Omega}\right) \mathcal{H}_{\mathcal{H}}(\mathbf{x})\right) 1_{\Omega}(\mathbf{x})$, where we recall Definition 4.3 . Then the translation covariant multi-scale Helmholtz decomposition of $\tilde{\mathbf{v}}$ is given by

$$
\left(\phi_{s} * \tilde{\mathbf{v}}\right)(\mathbf{x})=\sum_{i=1}^{2}\left(\mathbf{k}_{i}^{\mathrm{rf}, s} * \widetilde{v}^{i}\right)(\mathbf{x})+\sum_{i=1}^{2}\left(\mathbf{k}_{i}^{\mathrm{df}, s} * \widetilde{v}^{i}\right)(\mathbf{x}),
$$

where the analytic multi-scale rotation-free $\operatorname{kernel} \mathbf{k}_{i}^{\mathrm{rf}, s}$ is given by

$$
\begin{aligned}
& \mathbf{k}_{1}^{\mathrm{rf}, s}(x, y)=\frac{e^{-\frac{x^{2}+y^{2}}{4 s}}}{4 \pi s\left(x^{2}+y^{2}\right)^{2}}\left(\begin{array}{l}
-2\left(e^{\frac{x^{2}+y^{2}}{4 s}}-1\right) s\left(x^{2}-y^{2}\right)+x^{2}\left(x^{2}+y^{2}\right) \\
-4 x y\left(e^{\frac{x^{2}+y^{2}}{4 s}}-1\right) s+x y\left(x^{2}+y^{2}\right)
\end{array}\right), \\
& \mathbf{k}_{2}^{\mathrm{rf}, s}(x, y)=\frac{e^{-\frac{x^{2}+y^{2}}{4 s}}}{4 \pi s\left(x^{2}+y^{2}\right)^{2}}\left(\begin{array}{l}
-4 x y\left(e^{\frac{x^{2}+y^{2}}{4 s}}-1\right) s+x y\left(x^{2}+y^{2}\right) \\
2\left(e^{\frac{x^{2}+y^{2}}{4 s}}-1\right) s\left(x^{2}-y^{2}\right)+y^{2}\left(x^{2}+y^{2}\right)
\end{array}\right) .
\end{aligned}
$$

The analytic multi-scale divergence-free kernel $\mathbf{k}_{i}^{\mathrm{df}, s}$ is given by

$$
\mathbf{k}_{1}^{\mathrm{df}, s}=\left(\phi_{s}-k_{1}^{\mathrm{rf}, s, 1},-k_{1}^{\mathrm{rf}, s, 2}\right)^{T} \text { and } \mathbf{k}_{2}^{\mathrm{df}, s}=\left(-k_{2}^{\mathrm{rf}, s, 1},-k_{2}^{\mathrm{rf}, s, 2}+\phi_{s}\right)^{T} .
$$


Proof. Set $G_{s}^{2 D}=\phi_{s} * G^{2 D}$, where $\phi_{s}$ denotes the heat kernel given in (3.1) and $G^{2 D}$ denotes the Green's function given in (4.7). The diffused first-order derivative (with respect to $x$ ) of the Green's function can be computed via:

$$
\begin{aligned}
\partial_{x} G_{s}^{2 D}(\mathbf{x}) & =\mathcal{F}^{-1}\left(\left(\omega_{1}, \omega_{2}\right) \mapsto \frac{i \omega_{1}}{2 \pi} \int_{s}^{\infty} \exp \left(-t\left(\omega_{1}^{2}+\omega_{2}^{2}\right)\right) \mathrm{d} t\right)(x, y) \\
& =\int_{s}^{\infty} \frac{x \exp \left(-\frac{x^{2}+y^{2}}{4 t}\right)}{8 \pi t^{2}} \mathrm{~d} t=\frac{x}{2 \pi} \frac{1-\exp \left(-\frac{x^{2}+y^{2}}{4 s}\right)}{x^{2}+y^{2}}, \quad \mathbf{x}=(x, y),
\end{aligned}
$$

where $\omega_{1}$ and $\omega_{2}$ denote the frequency variables; cf. [8, Eq.14]. Similarly, one has

$$
\partial_{y} G_{s}^{2 D}(\mathbf{x})=\frac{y}{2 \pi} \frac{1-\exp \left(-\frac{x^{2}+y^{2}}{4 s}\right)}{x^{2}+y^{2}} .
$$

The Helmholtz decomposition commutes with the diffusion operator, and we express both the divergence and gradient operator by Gaussian derivatives in (4.10), where we can exploit the semigroup property of diffusion: $\phi_{s}=\phi_{\frac{s}{2}} * \phi_{\frac{s}{2}}$. For the rotation-free part we get

$$
\begin{aligned}
\nabla^{\left(\frac{s}{2}\right)} \operatorname{div}\left(\frac{s}{2}\right) \mathcal{G} \widetilde{\mathbf{v}} & =\sum_{i=1}^{2}\left(\nabla \partial_{x^{i}} G_{2 D}^{s} * \widetilde{v}^{i}\right)(\mathbf{x}) \\
& =\sum_{i=1}^{2}\left(\mathbf{k}_{i}^{\mathrm{rf}, s} * \widetilde{v}^{i}\right)(\mathbf{x}):=\sum_{i=1}^{2}\left(\left(k_{i}^{\mathrm{rf}, s, 1} * \widetilde{v}^{i}\right)(\mathbf{x}),\left(k_{i}^{\mathrm{rf}, s, 2} * \widetilde{v}^{i}\right)(\mathbf{x})\right)^{T},
\end{aligned}
$$

with $x^{1}=x, x^{2}=y$, with $\mathbf{k}_{i}^{\mathrm{rf}, s}=\nabla \partial_{x^{i}} G_{2 D}^{s}$ and where e.g. $\nabla^{\left(\frac{s}{2}\right)}$ denotes the Gaussian gradient at scale $\frac{s}{2}$, i.e. $\nabla^{\left(\frac{s}{2}\right)} f=\nabla\left(\phi_{\frac{s}{2}} * f\right)=\left(\nabla \phi_{\frac{s}{2}} * f\right)$. Finally, regarding the identity (4.13) we note that $\phi_{s} * \tilde{\mathbf{v}}=\nabla^{\left(\frac{s}{2}\right)} \operatorname{div}\left(\frac{s}{2}\right) \mathcal{G} \tilde{\mathbf{v}}-\widetilde{\operatorname{rot}}{ }^{\left(\frac{s}{2}\right)} \operatorname{rot}\left(\frac{s}{2}\right) \mathcal{G} \tilde{\mathbf{v}}$.

REMARK 4.6. In our algorithm the kernels $\mathbf{k}_{i}^{\mathrm{rf}, s}$ and $\mathbf{k}_{i}^{\mathrm{df}, s}$ are analytically precomputed for a range of scales (according to the scale of the involved velocity features; cf. Section 3) via (4.12) and (4.13). The diffusion takes care of the singularities present at $s=0$ : The kernels $\mathbf{k}_{i}^{\mathrm{rf}, s}$ and $\mathbf{k}_{i}^{\mathrm{df}, s}$ are nonsingular iff $s>0$.
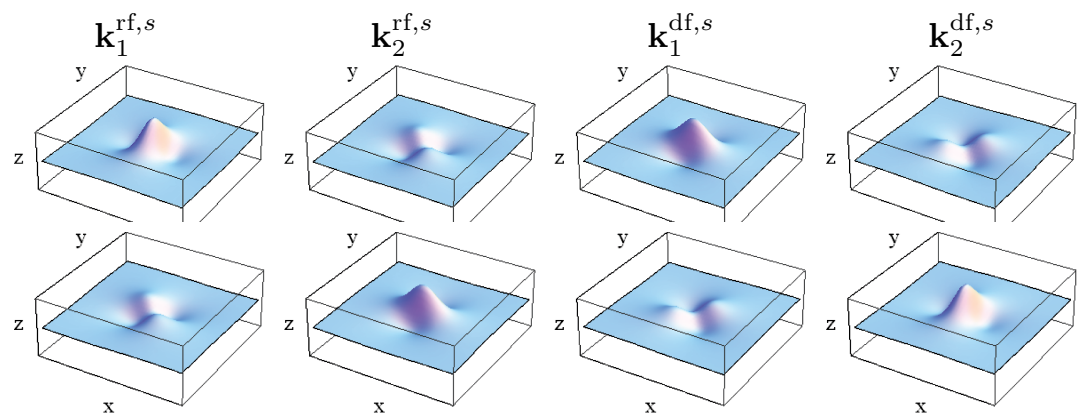

FIG. 6. Effective kernels of the multi-scale Helmholtz decomposition (4.11) at a fixed scale $s>0$. Top row: first component of respectively from left to right $\mathbf{k}_{1}^{\mathrm{rf}, s}, \mathbf{k}_{2}^{\mathrm{rf}, s}, \mathbf{k}_{1}^{\mathrm{df}, s}, \mathbf{k}_{2}^{\mathrm{df}, s}$. Bottom row: second component of respectively from left to right $\mathbf{k}_{1}^{\mathrm{rf}, s}, \mathbf{k}_{2}^{\mathrm{rf}, s}, \mathbf{k}_{1}^{\mathrm{df}, s}, \mathbf{k}_{2}^{\mathrm{df}, s}$. 
5. A brief motivation for using covariant derivatives. Usually one computes the derivative of a gray-value image $f: \Omega \rightarrow \mathbb{R}$ by means of a Gaussian derivative

$$
\partial_{x}^{(s)} f=\partial_{x}\left(\phi_{s} * f\right)=\left(\partial_{x} \phi_{s}\right) * f
$$

or by a finite difference (i.e., replace first-order Gaussian by the discrete $[1,-1]$-stencil). The local slope, say in the $x$-direction, of the graph at $(\mathbf{x}, f(\mathbf{x}))$ measured by $\partial_{x}^{(s)}(f)(\mathbf{x})$ is independent of the local height $f(\mathbf{x})$. Visual perception, however, does not work like this. Consider for example Figure 7. The slope in the dark areas is often perceived differently than the slope in the light areas. This remarkable property of visual perception can be modeled by replacing the derivative/gradient by a covariant derivative in a vector bundle, where sections coincide with the graph of the functions.

According to [23, 33, 17] the covariant derivative of a differentiable gray-value image $f: \mathbb{R}^{2} \rightarrow \mathbb{R}$ is given by

$$
D^{h} f(x, y)=D f(x, y)-\frac{1}{h(x, y)} D h(x, y) f(x, y)
$$

for all points $(x, y) \in \Omega \subset \mathbb{R}^{2}$ where $h(x, y) \neq 0$. The covariant derivative is invariant under scalar multiplication of the gauge function since

$$
D^{\lambda h} f=D^{|h|} f=D^{h} f \text {, for all } h \neq 0 .
$$

To get an impression of the use of covariant derivatives, see Figure 7 (top left figure). As the rectangle has constant brightness (say $f(x, y)=1$ ) the visual perception of a gradient cannot be explained using standard derivatives, since the regular gradient vanishes within the rectangle. But if we define gauge functions as indicated by white dashed boxes in the image, i.e. $h_{i}(\tilde{x}, \tilde{y})=\tilde{x}-x+C_{i}$ for all positions $(\tilde{x}, \tilde{y}) \in \mathbb{R}^{2}$ with respect to the origin in the middle of the $i$ th dashed rectangle, with $C_{i}>0$, then at position $(x, y)$ within the black rectangle we have $D^{h_{i}} f(x, y)=D f(x, y)-\frac{D h_{i}(x, y)}{h_{i}(x, y)} f(x, y)=\left(-\frac{1}{C_{i}}, 0\right)$. Black vectors indicate covariant gradients whereas white vectors indicate regular gradients. The gauge function index $i$ is indicated by different dashing.

6. Covariant derivatives. Next we extend the concept of covariant derivatives to covariant derivatives of motion fields. To this end we consider the vector bundle

$$
E:=\left(\Omega \times \mathbb{R}^{2}, \pi, \Omega\right),
$$

where $\Omega \subset \mathbb{R}^{2}$ is the image domain and where the fundamental projection $\pi: \Omega \times \mathbb{R}^{2} \rightarrow \Omega$ is given by

$$
\pi\left(x, y, v^{1}, v^{2}\right)=(x, y), \quad\left(x, y, v^{1}, v^{2}\right) \in \Omega \times \mathbb{R},
$$

where respectively $v^{1}$ and $v^{2}$ denote velocity in the $x$ and $y$ directions.

A fiber in this vector bundle is the two-dimensional vector space

$$
\pi^{-1}(x, y)=\left\{\left(x, y, v^{1}, v^{2}\right) \mid v^{1}, v^{2} \in \mathbb{R}\right\} .
$$

A section $\sigma$ in the vector bundle is the surface which basically represents the graph of some vector-valued function $v: \Omega \rightarrow \mathbb{R}^{2}$ :

$$
\sigma_{\mathbf{v}}(x, y)=\left\{\left(x, y, v^{1}, v^{2}\right) \in \Omega \times \mathbb{R}^{2} \mid v^{1}=v^{1}(x, y), v^{2}=v^{2}(x, y)\right\}, \quad \mathbf{v}=\left(v^{1}, v^{2}\right)^{T} ;
$$

note that $\pi \circ \sigma_{\mathbf{v}}=\operatorname{id}_{\Omega}$, i.e. $\left(\pi \circ \sigma_{\mathbf{v}}\right)(x, y)=(x, y)$ for all $(x, y) \in \Omega$. 


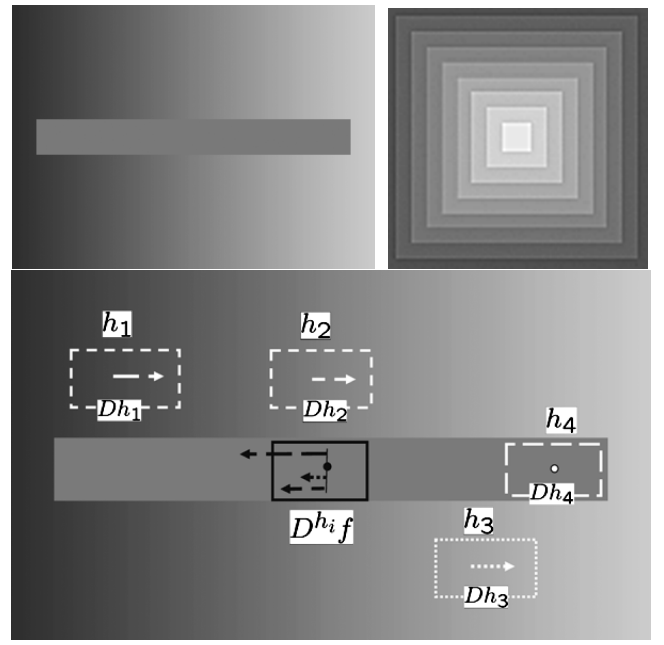

FIG. 7. Top row: The visual illusion on the left illustrates that due to surrounding gray values a slope is perceived in the rectangle, although the rectangle has constant brightness. The visual illusion on the right illustrates the opposite dependence: due to different surrounding slopes, the same brightness is perceived differently (the diagonals appear brighter). Bottom row: The left visual illusion can be modeled by covariant derivatives.

6.1. Connections on the Vector Bundle E. A connection on a vector bundle is by definition a mapping $D: \Gamma(E) \rightarrow \mathcal{L}(\Gamma(T(\Omega)), \Gamma(E))$ from the space of sections in the vector bundle $\Gamma(E)$ to the space of linear mappings $\mathcal{L}(\Gamma(T(\Omega)), \Gamma(E))$ from the space of vector fields on $\Omega$ denoted by $\Gamma(T(\Omega))$ into the space of sections $\Gamma(E)$ in the vector bundle $E$ such that

$$
\begin{array}{ll}
D_{\mathbf{v}+\mathbf{w}} \sigma=D_{\mathbf{v}} \sigma+D_{\mathbf{w}} \sigma, & D_{f \mathbf{v}} \sigma=f D_{\mathbf{v}} \sigma, \\
D_{\mathbf{v}}(\sigma+\tau)=D_{\mathbf{v}} \sigma+D_{\mathbf{v}} \tau, & D_{\mathbf{v}}(f \sigma)=\mathbf{v}(f) \sigma+f D_{\mathbf{v}} \sigma
\end{array}
$$

for all vector fields $\mathbf{v}=\sum_{i=1}^{2} v^{i} \partial_{x^{i}}, \mathbf{w}=\sum_{i=1}^{2} w^{i} \partial_{x^{i}} \in \Gamma(T(\Omega)$ ) (i.e., sections in the tangent bundle $T(\Omega))$ and all $f \in C^{\infty}(\Omega, \mathbb{R})$ and all sections $\sigma \in \Gamma(E)$ in the vector bundle $E$. We used the common short notation $D_{\mathbf{v}} \sigma=(D \sigma)(\mathbf{v})$. Now (6.2) implies that

$$
\begin{aligned}
\left(\left(D \sigma_{\mathbf{v}}\right)(X)\right)(\mathbf{c}(t)) & =D\left(v^{1} \sigma_{1}+v^{2} \sigma_{2}\right)(X)(\mathbf{c}(t)) \\
& =\left.\sum_{j=1}^{2} X\right|_{\mathbf{c}(t)}\left(v^{j}\right) \sigma_{j}+\sum_{j=1}^{2} \sum_{i=1}^{2} v^{j}(\mathbf{c}(t)) \dot{c}^{i}(t)\left(D \partial_{x^{i}} \sigma_{j}\right)(\mathbf{c}(t)),
\end{aligned}
$$

where $\sigma_{1}(x, y)=(x, y, 1,0)$ and $\sigma_{2}(x, y)=(x, y, 0,1)$ denote the unit sections in the $x$ and $y$ directions and where $\left.X\right|_{\mathbf{c}(t)}=\left.\sum_{i=1}^{2} \dot{c}^{i}(t) \partial_{x^{i}}\right|_{\mathbf{c}(t)}$ denotes a vector field on $\Omega$ tangent to a curve $\mathbf{c}:(0,1) \rightarrow \Omega$ in the image domain $\Omega \subset \mathbb{R}^{2}$.

By (6.3) the connection $D$ is uniquely determined by $\left\{D_{\partial_{x^{i}}} \sigma_{j}\right\}_{i, j=1,2}$. Now for each $i, j=1,2$ this output $D_{\partial_{x^{i}}} \sigma_{j}$ is a section and consequently there exist unique functions $\Gamma_{i j}^{k}: \Omega \rightarrow \mathbb{R}$ (Christoffel symbols) such that $\left(D_{\partial_{x^{i}}} \sigma_{j}\right)(\mathbf{c}(t))=\sum_{k=1}^{2} \Gamma_{i j}^{k}(\mathbf{c}(t)) \sigma_{k}$. Here we 
restrict ourselves to the diagonal case w.r.t. Euclidean coordinates

$$
\Gamma_{i j}^{k}=A_{i}^{j} \delta_{j}^{k}, \text { with } A_{i}^{k}:=\Gamma_{i k}^{k} .
$$

We impose this restriction for pragmatic reasons: It is a straightforward generalization of our previous work on reconstruction of scalar-valued functions using covariant derivatives [33, although this choice does not affect the rules for covariant derivatives on a vector bundle (6.2). However, when using such derivatives in a velocity reconstruction, we will have to check the rotation covariance of the algorithm.

6.2. Covariant derivatives on the vector bundle $E$ induced by gauge fields. By our restriction (6.4) the covariant derivatives in $E$ are given by

$$
D_{\partial x^{i}} \sigma_{\mathbf{v}}=\left(D \sigma_{\mathbf{v}}\right)\left(\partial_{x^{i}}\right)=\left(\partial_{x^{i}} v^{1}+A_{i}^{1} v^{1}\right) \sigma_{1}+\left(\partial_{x^{i}} v^{2}+A_{i}^{2} v^{2}\right) \sigma_{2}
$$

with $\mathbf{v}=\sum_{i=1}^{2} v^{i} \sigma_{i} \in \Gamma(E)$. Now we choose $\left\{A_{i}^{j}\right\}$ such that an a priori given section $\sigma_{\mathbf{h}}(\mathrm{a}$ so-called gauge field, 33] $)(x, y) \mapsto \sigma_{\mathbf{h}}(x, y)$ with $\sigma_{\mathbf{h}}(x, y)=\left(x, y, h^{1}(x, y), h^{2}(x, y)\right), \mathbf{h}=$ $\left(h^{1}, h^{2}\right)^{T}$, is not affected ("invisible") by the covariant derivative; i.e., we must solve for

$$
\begin{aligned}
& \left(D \sigma_{\mathbf{h}}\right)\left(\partial_{x^{i}}\right)=0 \Leftrightarrow\left(\partial_{x^{i}} h^{1}+A_{i}^{1} h^{1}\right) \sigma_{1}+\left(\partial_{x^{i}} h^{2}+A_{i}^{2} h^{2}\right) \sigma_{2}=0 \sigma_{1}+0 \sigma_{2} \\
& \Leftrightarrow A_{i}^{j}=-\frac{\partial_{x^{i}} h^{j}}{h^{j}}, \text { for all } i, j=1,2,
\end{aligned}
$$

so that the covariant derivative $D^{\mathbf{h}}$ induced by the gauge field $\sigma_{\mathbf{h}} \in \Gamma(E)$ is given by

$$
\left(D_{\partial x^{i}}^{\mathbf{h}} \sigma_{\mathbf{v}}\right)(\mathbf{x})=\left(\left(\partial_{x^{i}}\right)^{h_{1}} v^{1}\right)(\mathbf{x}) \sigma_{1}+\left(\left(\partial_{x^{i}}\right)^{h_{2}} v^{2}\right)(\mathbf{x}) \sigma_{2},
$$

where we use the short notation

$$
\left(\partial_{x^{i}}\right)^{h^{j}} v^{j}=\frac{\partial v^{j}}{\partial x^{i}}-\frac{\frac{\partial h^{j}}{\partial x^{i}}}{h^{j}} v^{j}, \quad \text { for } j=1,2 .
$$

See Figure 8 for a geometrical interpretation of covariant derivatives, where for the sake of illustration we consider the subset $E_{0}:=\left\{\left(x, y, v^{1}, v^{2}\right) \in E \mid v^{2}=0\right\}$ of the total vector bundle consisting of all elements for which the fourth component vanishes and where both the gauge field $\sigma_{\mathbf{h}}$ and the velocity field $\sigma_{\mathbf{v}}$ are of the form $\sigma_{\mathbf{v}}(x, y)=(x, y, f(x), 0)$.

REMARK 6.1. We consider the vector bundle $E:=\left(\Omega \times \mathbb{R}^{2}, \pi, \Omega\right)$ with connection $D$. One could also consider a tangent bundle $(\Omega, T(\Omega))$ equipped with a Levi-Cevita connection $\nabla$ induced by some (image-dependent) metric $g$. These connections could be combined in a tensor product $\nabla \otimes D$ which requires cumbersome bookkeeping. In [17] they are combined via new connection components $\mathcal{D}_{m} v^{j}=\left(\left(\partial_{m}+A_{m}^{i}\right) \delta_{i}^{j}+\tilde{\Gamma}_{i m}^{j}\right) v^{i}$, where $\tilde{\Gamma}_{m i}^{j}$ denote Christoffel symbols w.r.t. $\nabla$ and where the flat case corresponds to (6.4). In any case (flat or nonflat $\nabla$ ) we need to check whether the overall velocity reconstruction algorithm described in Figure 2 is covariant with respect to rotations and translations acting simultaneously on the reconstructed field and the gauge field, as this is not a priori guaranteed.

Now that we have introduced everything in a formal differential geometry setting we will simplify our notation. In the remainder of this article, we will identify sections $\sigma_{\mathbf{v}}$ in $E$ with the corresponding vector functions $\mathbf{v}: \Omega \rightarrow \mathbb{R}^{2}$,

$$
\begin{array}{ll}
\sigma_{\mathbf{v}=v^{1}, v^{2}}(\mathbf{x})=\left(\mathbf{x}, v^{1}(\mathbf{x}), v^{2}(\mathbf{x})\right) & \leftrightarrow \mathbf{v}(\mathbf{x})=\left(v^{1}(\mathbf{x}), v^{2}(\mathbf{x})\right)^{T}, \text { for all } \mathbf{x} \in \mathbb{R}^{2}, \\
\sigma_{1}=(0,0,1,0) & \leftrightarrow \mathbf{e}_{1}:=(1,0)^{T}, \\
\sigma_{2}=(0,0,0,1) & \leftrightarrow \mathbf{e}_{2}:=(0,1)^{T},
\end{array}
$$




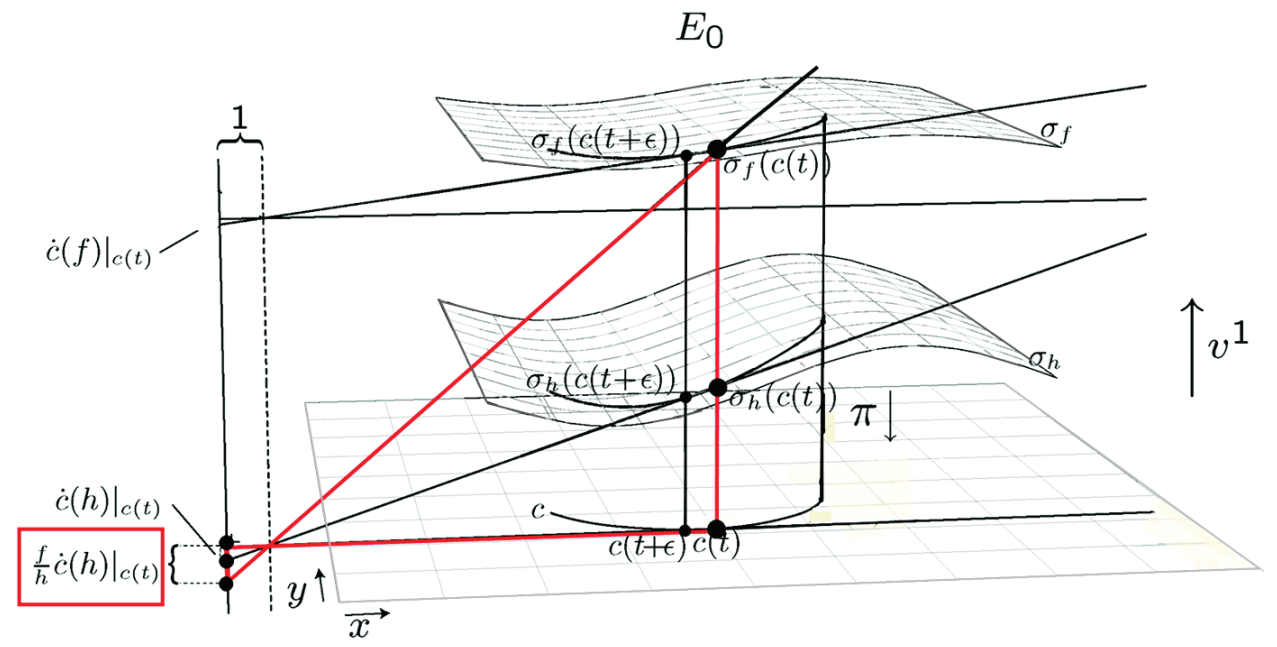

FIG. 8. Top row: Consider the point $\sigma_{\mathbf{v}}(c(t))=(c(t), f(c(t)), 0)$ in the vector bundle $E_{0} \subset E$, where $c$ denotes a curve in the domain $\Omega \subset$ $\mathbb{R}^{2}$. Consider the corresponding point $\sigma_{\mathbf{h}}(c(t))=(c(t), h(c(t)), 0)$ on the gauge field section and the base point $c(t) \in \Omega$. The directional derivative of this point in direction $\dot{c}(t)$ equals $\dot{c}(f):=D f(c(t)) \cdot \dot{c}(t)$ and it is slightly positive. The covariant derivative at this point is $D^{\mathbf{h}} \sigma_{\mathbf{v}}(c(t))=\dot{c}(f)-\frac{f(c(t))}{h(c(t))} \dot{c}(h)$ and is negative. The geometrical congruency underlying the extra term in a covariant derivative in vector bundle $E$ is indicated in red.

and write $\partial_{x^{i}}^{\mathbf{h}} \mathbf{v}: \Omega \rightarrow \mathbb{R}^{2}$ for the vector function corresponding to the section $D_{\partial x^{i}}^{\mathbf{h}} \sigma_{\mathbf{v}}$ : $\Omega \rightarrow E$, i.e. :

$$
\left(x, y, \partial_{x^{i}}^{\mathbf{h}} \mathbf{v}(x, y)\right)=\left(x, y, \partial_{x^{i}}^{h^{1}} v^{1}, \partial_{x^{i}}^{h^{2}} v^{2}\right)=\left(D_{\partial x^{i}}^{\mathbf{h}} \sigma_{\mathbf{v}}\right)(x, y),
$$

where we applied the short notation in (6.7). Note that covariant derivatives are invariant under sign transitions; recall (5.2). The covariant Laplacian can be explicitly expressed in the components

$$
\left(D^{\mathbf{h}}\right)^{*} D^{\mathbf{h}} \mathbf{v}=\sum_{j=1}^{2} \sum_{i=1}^{2}\left(\left(\partial_{x^{i}}^{h^{j}}\right)^{*} \partial_{x^{i}}^{h^{j}} v^{j}\right) \mathbf{e}_{j}=\sum_{j=1}^{2}\left(-\Delta v^{j}+\frac{\Delta h^{j}}{h^{j}} v^{j}\right) \mathbf{e}_{j}
$$

where we recall our identifications (6.8). The $\mathbb{L}_{2}$-adjoint of the covariant derivative, (6.7), is defined (on the same domain) by

$$
\left(\left(\partial_{x^{i}}\right)^{h^{j}} \phi, v^{j}\right)_{\mathbb{L}_{2}(\Omega)}=\left(\phi,\left(\left(\partial_{x^{i}}\right)^{h^{j}}\right)^{*} v^{j}\right)_{\mathbb{L}_{2}(\Omega)} \text { for all } \phi, v^{j} \in \mathbb{H}_{1}(\Omega) .
$$

Integration by parts yields

$$
\left(\left(\partial_{x^{i}}\right)^{h^{j}}\right)^{*} v^{j}=-\frac{\partial v^{j}}{\partial x^{i}}-\frac{\frac{\partial h^{j}}{\partial x^{i}}}{h^{j}} v^{j} .
$$

If we compare the adjoint covariant derivative to the covariant derivative we see that the multiplicator part is maintained whereas the derivative part contains an extra minus 
sign, cf. [17, that is missing in [23, Eq. 21]. By straightforward computation one finds the fundamental formula:

$$
\begin{aligned}
-\Delta^{h^{j}} v^{j}:=\sum_{i=1}^{2}\left(\partial_{x^{i}}^{h^{j}}\right)^{*} \partial_{x^{i}}^{h^{j}} v^{j} & =\sum_{i=1}^{2}-\frac{\partial}{\partial x^{i}}\left(\frac{\partial}{\partial x^{i}}\right)^{h^{j}} v^{j}-\frac{\frac{\partial h^{j}}{\partial x^{i}}\left(\frac{\partial}{\partial x^{i}}\right)^{h^{j}} v^{j}}{h^{j}} \\
& =\sum_{i=1}^{2}-\frac{\partial}{\partial x^{i}}\left(\frac{\partial v^{j}}{\partial x^{i}}-\frac{\partial h^{j}}{\partial x^{i}} \frac{v^{j}}{h^{j}}\right)-\frac{\frac{\partial v^{j}}{\partial x^{i}}\left(\frac{\partial v^{j}}{\partial x^{i}}-\frac{\partial h^{j}}{\partial x^{i}} \frac{v^{j}}{h h^{j}}\right)}{h^{j}} \\
& =-\Delta v^{j}+\frac{\Delta h^{j}}{h^{j}} v^{j} .
\end{aligned}
$$

Now that we have introduced the covariant Laplacian, we mention two preliminary issues that directly arise from (6.11) and which will be addressed in the remainder of this article.

REMARK 6.2. At first sight the covariant derivatives and their associated (inverse) Laplacian seem to be ill-posed as the gauge-field components should not vanish. However, the crucial scaling property of covariant derivatives, Eq. (5.2), allows us to scale away from 0. Furthermore, as we will see later in Section 7 the Dirichlet kernel operator of the coercive covariant Laplacian is stable. Finally, we will show how the manifest stability of our algorithms depends on the choice of the gauge field.

6.3. Interpolation between conventional derivatives and covariant derivatives. A monotonic transformation on the components of the gauge field takes care of the interpolation between standard derivatives and covariant derivatives. For the sake of illustration we restrict ourselves to the scalar-valued case (with positive gauge function $h: \Omega \rightarrow \mathbb{R}^{+}$, recall (5.2) ) as the vector-valued case follows by applying everything on the two separate components. By applying a monotonic transformation $h \mapsto h^{\eta}$ on the gauge function we obtain the following covariant derivative:

$$
D^{h^{\eta}} f=D f-D\left(\log h^{\eta}\right) f=D f-\eta(D \log h) f .
$$

If $\eta=0$ the expression (6.12) provides a conventional derivative, whereas the case $\eta=1$ yields a covariant derivative with respect to the gauge function $h$. For further motivation and experiments on the choice of $\eta$ we refer to our technical report [12, ch:10, Fig.15].

7. Fundamental properties of the selfadjoint covariant Laplacian. In this section we shall show that the covariant Laplacian has more or less the same fundamental properties as the ordinary Laplacian. These basic fundamental properties including selfadjointness, negative definiteness, and coercivity are important for well-posed symmetric inverse problems that shall arise from Euler-Lagrange equations for vector field interpolation later on in Section 9 and Section 11.

Definition 7.1. Let $H$ be a Hilbert space with inner product $(\cdot, \cdot)$. An unbounded operator $A: H \rightarrow H$ on a Hilbert space $H$ with domain $\mathcal{D}(A)$ is selfadjoint if

$$
(A f, g)=(f, A g) \text { for all } f, g \in \mathcal{D}(A),
$$

and if the domain of the adjoint $\mathcal{D}\left(A^{*}\right)$ coincides with the domain of $A$, i.e. $\mathcal{D}\left(A^{*}\right)=$ $\mathcal{D}(A)$. Such an operator is coercive if there exists a positive constant $c>0$ s.t.

$$
(A f, f) \geq c(f, f) \text { for all } f \in \mathcal{D}(A) .
$$


Now suppose that the gauge field is twice continuously differentiable and $\left|h^{j}\right|>0$. By (5.2) we can assume that the components $h^{j}$ of the gauge field are positive, $h^{j}>0$. The covariant Laplacian $\Delta^{h^{j}}:=-\sum_{i=1,2}\left(\partial_{x^{i}}^{h^{j}}\right)^{*} \partial_{x^{i}}^{h^{j}}$ is just like the ordinary Laplacian, an unbounded negative definite operator on $\mathbb{L}_{2}(\Omega)$ with domain

$$
\mathcal{D}\left(-\Delta^{h^{j}}\right)=\mathbb{H}_{2}^{0}(\Omega):=\left\{f \in \mathbb{H}_{2}(\Omega):\left.f\right|_{\partial \Omega}=0\right\} .
$$

The covariant Laplacian operator $\Delta^{h^{j}}$ is negative definite

$$
\left(-\Delta^{h^{j}} f, f\right)_{\mathbb{L}_{2}(\Omega)}=\left(\partial_{x^{i}}^{h^{j}} f, \partial_{x^{i}}^{h^{j}} f\right)_{\mathbb{L}_{2}(\Omega)}>0
$$

for all $j=1,2$ and all $f \neq 0$ regardless of the choice of $h^{j}$. The functions $q^{j}:=\frac{\Delta h^{j}}{h^{j}}$ are continuous on the compact domain $\Omega$, so there exists some $\mathbf{x}_{0}^{j}, \mathbf{y}_{0}^{j} \in \Omega$ such that

$$
\frac{\Delta h^{j}\left(\mathbf{y}_{0}^{j}\right)}{h^{j}\left(\mathbf{y}_{0}^{j}\right)} \leq q^{j}:=\frac{\Delta h^{j}}{h^{j}} \leq \frac{\Delta h^{j}\left(\mathbf{x}_{0}^{j}\right)}{h^{j}\left(\mathbf{x}_{0}^{j}\right)} .
$$

Now minus the covariant Laplacian is a Sturm-Liouville operator 28]. Consequently, the covariant Laplacian is selfadjoint, and the corresponding selfadjoint resolvent operator

$$
\left(I-\Delta^{h^{j}}\right)^{-1}
$$

is compact, and thereby there exists a complete orthonormal set of strictly positive eigenvalues and eigenfunctions [50, Thm. 13.33] such that

$$
\left\{\begin{array} { r l } 
{ - \Delta ^ { h ^ { j } } f _ { n } ^ { j } } & { = \lambda _ { n } ^ { j } f _ { n } ^ { j } , j = 1 , 2 , } \\
{ f _ { n } ^ { j } | _ { \partial \Omega } } & { = 0 , j = 1 , 2 , }
\end{array} \Leftrightarrow \left\{\begin{array}{rl}
-\Delta\left(f_{n}^{j}\right)+q^{j} f_{n}^{j} & =\lambda_{n}^{j} f_{n}^{j}, j=1,2, \\
\left.f_{n}^{j}\right|_{\partial \Omega} & =0, j=1,2,
\end{array}\right.\right.
$$

where we stress that $\lambda_{n}=0$ would yield the trivial solution only, as $D^{h^{j}} f^{j}=0$ implies $f^{j}=\lambda h^{j}$ which for $\lambda \neq 0$ would contradict (7.2) since $\left.f_{n}^{j}\right|_{\partial \Omega}=0$. Now the resolvent of the covariant Laplacian is compact with a domain $\mathbb{H}_{2}^{0}(\Omega)$ that is compactly embedded in $\mathbb{L}_{2}(\Omega)$, and, consequently, 0 is the only density point of the spectrum of the resolvent. Consequently, the spectrum of the minus covariant Laplacian is contained in

$$
\sigma\left(-\Delta^{h^{j}}\right) \subset\left[c_{h}(\Omega), \infty\right)
$$

for some $c_{h}(\Omega)>0$ and by the Sturm-Liouville theory [25] the spectrum only consists of eigenvalues so that $c_{h}(\Omega)$ equals the smallest eigenvalue $\lambda_{1}^{j}$ of $-\Delta^{h^{j}}$ restricted to its domain (7.1) which can be expressed by the Rayleigh quotient

$$
\lambda_{1}^{j}=\min _{f \in \mathbb{H}_{2}^{0}(\Omega)} \frac{\int_{\Omega}-f(\mathbf{x}) \Delta f(\mathbf{x})+q^{j}(\mathbf{x})|f(\mathbf{x})|^{2} \mathrm{~d} \mathbf{x}}{\int_{\Omega}|f|^{2}(\mathbf{x}) \mathrm{d} \mathbf{x}}=\min _{f \in \mathbb{H}_{2}^{0}(\Omega)} \frac{\int_{\Omega}(\nabla f \cdot \nabla f)(\mathbf{x})+q^{j}(\mathbf{x})|f(\mathbf{x})|^{2} \mathrm{~d} \mathbf{x}}{\int_{\Omega}|f(\mathbf{x})|^{2} \mathrm{~d} \mathbf{x}} .
$$

We conclude that the covariant Laplacian is just like the regular Laplacian, a coercive operator on the domain $\mathbb{H}_{2}^{0}(\Omega)$ with a complete orthogonal basis of eigenfunctions. This coercivity is important for the stability of the numerical algorithms (via the Lax-Milgram theorem, 45]) later on, since inverting the covariant Laplacian boils down to inverting all the eigenvalues. In general it is apparent, from the essential formula (6.11), that the more convex the gauge field, the more well-posed the inversion of the covariant Laplacian and the covariant resolvent (7.3) is. See Example 7.2 and Example 7.3 below, 
where we respectively consider basic gauge functions that are convex and concave. As the covariant Laplacian (6.11) of a vector field acts componentwise, we will consider examples of a covariant Laplacian

$$
-\Delta^{h} f=\left(D^{h}\right)^{*} D^{h} f=-\Delta f+\frac{\Delta h}{h} f
$$

of a scalar field $f$ with respect to a scalar field $h$.

ExAmple 7.2. Consider the case where $\Omega=[0,1] \times[0,1]$ and $h(x, y)=\beta e^{\gamma_{1} x+\gamma_{2} y}$, with $\beta \in \mathbb{R}$ and $\beta \neq 0, \lambda_{i} \in \mathbb{R}$. Then

$$
\frac{\Delta h}{h}=|\gamma|^{2}=\left(\gamma_{1}\right)^{2}+\left(\gamma_{2}\right)^{2} \geq 0
$$

and we have $\left(D^{h}\right)^{*} D^{h}=-\Delta+|\gamma|^{2} I$. The smallest eigenvalue of the covariant Laplacian equals $2 \pi^{2}+\gamma^{2}$, which shows that the covariant Dirichlet problem is better posed than a regular Dirichlet problem in the case of a convex gauge function $h$.

EXAmple 7.3. Consider the case where $\Omega=[0,1] \times[0,1]$ and the gauge function is a Gaussian kernel $h(x, y)=\beta\left(\frac{1}{2 \pi \sigma^{2}} e^{-\frac{\left(x^{2}+y^{2}\right)}{2 \sigma^{2}}}\right)$, with $\beta>0$ arbitrary. Set $\alpha:=\frac{1}{2 \sigma^{2}}=\frac{3}{8}$. Then we end up with an extreme case where the gauge function is concave on the whole domain $\Omega$. A brief computation yields $q(x, y)=\frac{\Delta h(x, y)}{h(x, y)}=4 \alpha\left(\alpha\left(x^{2}+y^{2}\right)-1\right)$, and an application of the method of separation (where we set $f(x, y)=X_{m}(x) Y_{n}(y)$ ) to the eigenvalue problem $(-\Delta+q) f=\lambda_{m n} f$ with $\left.f\right|_{\partial \Omega}=0$ yields

$$
\mu_{1}^{m}+\mu_{2}^{n}-4 \alpha=\frac{4 \alpha^{2} x^{2} X_{m}(x)-X_{m}^{\prime \prime}(x)}{X_{m}(x)}-\frac{4 \alpha^{2} y^{2} Y_{n}(y)-Y_{n}^{\prime \prime}(y)}{Y_{n}(y)}-4 \alpha=\lambda_{m n}
$$

with $X_{m}(0)=X_{m}(1)=0$ and $Y_{n}(0)=Y_{n}(1)=0$ and where $\mu_{1}^{m}, \mu_{2}^{n}, \lambda_{m n}$ are separation constants, all defined by (7.4). So set $X_{m}(x)=e^{-\alpha x^{2}} \tilde{X}_{m}(2 \sqrt{\alpha} x)$ and $Y_{n}(y)=$ $e^{-\alpha y^{2}} \tilde{Y}_{n}(2 \sqrt{\alpha} y)$ and set $\xi=2 x \sqrt{\alpha}$ and $\eta=2 y \sqrt{\alpha}$. Then we arrive at the Hermite differential equation

$$
\begin{aligned}
& \tilde{X}_{m}^{\prime \prime}(\xi)-2 \xi \tilde{X}_{m}^{\prime}(\xi)-\frac{1}{2} \tilde{X}_{m}(\xi)=-\frac{\mu_{1}}{2 \alpha} \tilde{X}_{m}(\xi), \\
& \tilde{X}_{m}(0)=\tilde{X}_{m}(2 \sqrt{\alpha})=\tilde{X}_{m}\left(\sqrt{\frac{3}{2}}\right)=0
\end{aligned}
$$

and an analogous Hermite differential equation for $\tilde{Y}_{n}(\eta)$. Now we arrive at the Hermite polynomials $H_{m}(x)$ of order $m$, where the lowest order that could possibly fit the boundary conditions is $m=3$. This gives $\frac{\mu_{1}^{m}}{2 \alpha}-\frac{1}{2}=6=2 n \Rightarrow \mu_{1}^{m}=13 \alpha$ and the eigenfunction with smallest eigenvalue of the covariant Laplacian equals $(x, y) \mapsto$ $e^{-\alpha\left(x^{2}+y\right)^{2}} H_{3}(-2 \sqrt{\alpha} x) H_{3}(-2 \sqrt{\alpha} y)$ with eigenvalue $\lambda=\mu_{1}+\mu_{2}-4 \alpha=22 \alpha=8.25$, which is less than $2 \pi^{2}$ but still sufficiently far from 0 .

\section{The Green's function of the covariant Laplacian and an explicit coer-}

civity bound. In the general case, where $h \in C^{2}([0,1] \times[0,1], \mathbb{R})$ is arbitrary, we can get a grip on the coercivity constant $c_{h}(\Omega)$ by considering the Green's function of the covariant Laplacian. Here we employ the decomposition $-\Delta f+\frac{\Delta h}{h} f=\left(D^{h}\right)^{*} D^{h} f$ and we partially follow Sturm-Liouville theory, which does not directly apply since $h^{-1} \Delta h$ 
need not be positive. To obtain the coercivity constant for $\left(D^{h}\right)^{*} D^{h}$ we may restrict ourselves to the 1-dimensional case since

$$
\left(-\Delta+\frac{\Delta h}{h}\right) f=-\frac{\partial^{2} f}{\partial x^{2}}+\frac{\frac{\partial^{2} h}{\partial x^{2}}}{h} f-\frac{\partial^{2} f}{\partial y^{2}}+\frac{\frac{\partial^{2} h}{\partial y^{2}}}{h} f=\sum_{i=1}^{2}\left(\frac{\partial^{h}}{\partial x^{i}}\right)^{*}\left(\frac{\partial^{h}}{\partial x^{i}}\right) f .
$$

Thereby a lower bound $\tilde{c}_{\tilde{h}}([0,1])$ on the $1 \mathrm{D}$-covariant Laplacian,

$$
\mathbb{H}_{0}^{2}([0,1]) \ni \tilde{f} \mapsto D^{\tilde{h}} \tilde{f}=-\tilde{f}^{\prime \prime}+\frac{\tilde{h}^{\prime \prime}}{\tilde{h}} \tilde{f} \in \mathbb{L}_{2}([0,1]),
$$

produces a lower bound on the 2D-covariant Laplacian by means of the estimate

$$
\int_{0}^{1} \int_{0}^{1}\left|\frac{\partial^{h} f(x, y)}{\partial x}\right|^{2} \mathrm{~d} x \mathrm{~d} y \geq \int_{0}^{1} \tilde{c}_{h(\cdot, y)}([0,1]) \int_{0}^{1}|f(x, y)|^{2} \mathrm{~d} x \mathrm{~d} y .
$$

This lower bound on the spectrum of the $2 \mathrm{D}$ covariant Laplacian is given by

$$
\sigma\left(-\Delta+\frac{\Delta h}{h}\right)>c_{h}([0,1] \times[0,1]):=\min _{x \in[0,1]}\left\{\tilde{c}_{h(x, \cdot)}([0,1])\right\}+\min _{y \in[0,1]}\left\{\tilde{c}_{h(\cdot, y)}([0,1])\right\} .
$$

Therefore, in the remainder of this subsection and Appendix A, we consider a 1D gauge function $\tilde{h}:[0,1] \rightarrow \mathbb{R}$ and $\tilde{f} \in \mathbb{L}_{2}([0,1])$. For the sake of sober notation we omit the tildes and write $h \in C^{2}([0,1])$ with $h>0$ and $f \in \mathbb{L}_{2}([0,1])$.

Theorem 8.1. The Green's function of the 1D covariant Laplacian $f \mapsto\left(D^{h}\right)^{*} D^{h} f=$ $-f^{\prime \prime}+\frac{h^{\prime \prime}}{h} f$ is given by

$$
k_{h}(x, y)=\frac{h(x) h(y)}{Q_{h}(1)} \cdot \begin{cases}Q_{h}(x)\left(Q_{h}(1)-Q_{h}(y)\right) & \text { for } x \leq y \\ Q_{h}(y)\left(Q_{h}(1)-Q_{h}(x)\right) & \text { for } x>y\end{cases}
$$

with $Q_{h}(x):=\int_{0}^{x}(h(v))^{-2} \mathrm{~d} v$. The compact selfadjoint operator $K_{h}: \mathbb{L}_{2}([0,1]) \rightarrow$ $\mathbb{H}_{0}^{2}([0,1])$

$$
K_{h} f(x)=\int_{0}^{1} k_{h}(x, y) f(y) \mathrm{d} y
$$

is the right inverse of the covariant Laplacian

$$
\left(D^{h}\right)^{*} D^{h} K_{h} f=f \text { for all } f \in \mathbb{L}_{2}([0,1]) .
$$

The operator norm of $K_{h}$ can be estimated by the Hilbert-Schmidt norm

$$
\left\|K_{h}\right\| \leq\left\|\left|K_{h}\left\|\mid=\sqrt{\operatorname{trace} K_{h}^{*} K_{h}}=\right\| k_{h}\left\|_{\mathbb{L}_{2}\left([0,1]^{2}\right)} \leq \sqrt{2}\right\| h^{-1}\left\|_{\mathbb{L}_{2}([0,1])}\right\| h \|_{\mathbb{L}_{2}([0,1])},\right.\right.
$$

and we have the following lower bound for the spectrum of the 1D-covariant Laplacian

$$
\sigma\left(\left(D^{h}\right)^{*} D^{h}\right) \geq \frac{1}{\left\|K_{h}\right\| \|} \geq \tilde{c}_{h}([0,1]) \geq \frac{1}{\sqrt{2}}\left\|h^{-1}\right\|_{\mathbb{L}_{2}([0,1])}^{-1}\|h\|_{\mathbb{L}_{2}([0,1])}^{-1}
$$

and the following lower bound for the spectrum of the 2D-covariant Laplacian

$$
\begin{array}{r}
\sigma\left(-\Delta+\frac{\Delta h}{h}\right) \geq c_{h}([0,1] \times[0,1]) \\
\geq \frac{1}{\sqrt{2}} \min _{x \in[0,1]}\left\|h^{-1}(x, \cdot)\right\|_{\mathbb{L}_{2}([0,1])}\|h(x, \cdot)\|_{\mathbb{L}_{2}([0,1])} \\
+\frac{1}{\sqrt{2}} \min _{y \in[0,1]}\left\|h^{-1}(\cdot, y)\right\|_{\mathbb{L}_{2}([0,1])}\|h(\cdot, y)\|_{\mathbb{L}_{2}([0,1])} .
\end{array}
$$

The covariant Laplacian has a complete orthonormal basis of eigenfunctions, and the maximum lower bound $c_{h}([0,1] \times[0,1])$ coincides with the smallest eigenvalue. 
Proof. For the derivation of the main part of the theorem, that is, (8.2), (8.4) and inequality (8.6), we refer to Appendix A. Furthermore, we rely on standard results from functional analysis such as the fact that every Hilbert-Schmidt operator is compact, the Hilbert-Schmidt norm of a kernel operator $K_{h}$ equals the $\mathbb{L}_{2}$-norm of its kernel $h$, the spectral decomposition theorem for compact selfadjoint operators, and the fact that the Hilbert-Schmidt norm is an upper bound of the operator norm. Note that both operator $\left(D^{h}\right)^{*} D^{h}$ (unbounded on $\mathbb{L}_{2}([0,1])$ ) and operator $K_{h}$ are selfadjoint (with $\left.k_{h}(x, y)=k(y, x)\right)$ and by the spectral decomposition theorem for compact selfadjoint operators they share a common orthonormal basis of eigenfunctions from which the result follows. Finally, the lower-bound (8.7) follows by (8.6) and (8.1).

In Figure 9 we have plotted the graphs of Green's functions $x \mapsto k_{h}(x, y)$ of the covariant Laplacian for various gauge functions $h:[0,1] \rightarrow \mathbb{R}$ and several $y \in[0,1]$.
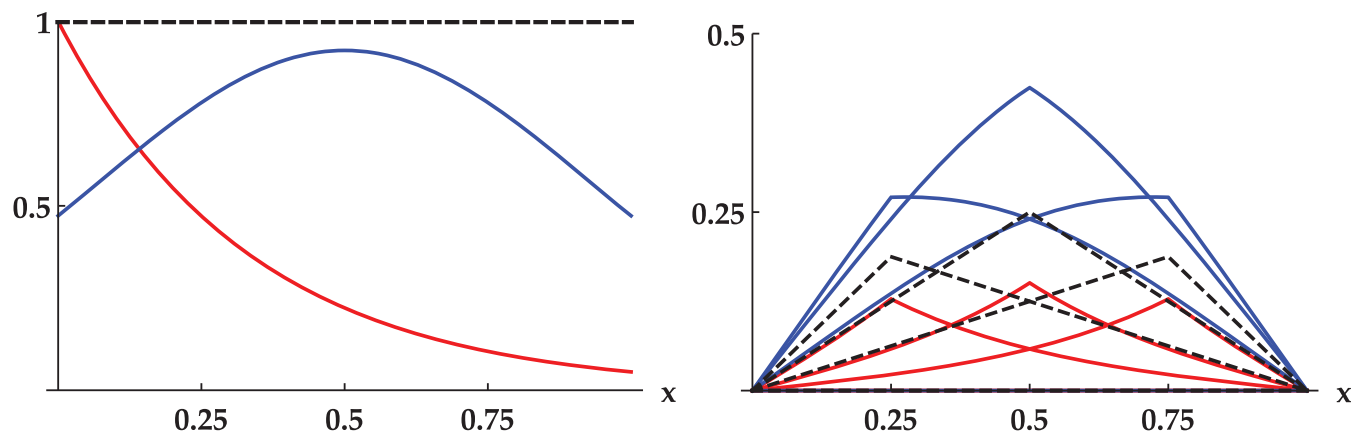

FIG. 9. The graphs of gauge functions $h$ and the corresponding Green's functions $k_{h}(\cdot, y)$ of the covariant Laplacian (where deltaspikes are respectively placed at $y=0.25,0.5,0.75)$. In red: gauge function of Example 7.2 (convex case, $\gamma=3$ ). In blue: gauge function of Example 7.3 (concave case, $\sigma=\frac{1}{4} \sqrt{3}$ ). In dashed black: Green's function with respect to constant gauge functions (i.e. Green's function of standard Laplacian). The Green's function is convex (concave) if the gauge field is convex (concave).

REMARK 8.2. The lower bounds in the estimates (8.6), (8.7) explicitly depend on the gauge field, but they need not be sharp. The invariance with respect to scaling of the gauge function

$$
\left(D^{h}\right)^{*} D^{h}=\left(D^{\lambda h}\right)^{*} D^{\lambda h} \text { and } K_{h}=K_{\lambda h} \text { and } c_{h}=c_{\lambda h}, \quad \lambda \neq 0,
$$

is also reflected in the estimate (8.7) as it should be.

Finally, we note the following result (which can also be observed in Figure 9).

LEMma 8.3. If the gauge function $h>0$ is convex (respectively concave), then the corresponding Green's function $k(\cdot, y)=\left(\left(D^{h}\right)^{*} D^{h}\right)^{-1} \delta_{y}$ of the covariant Laplacian is convex (respectively concave) as well.

For a proof of this result, see the last paragraph of Appendix A 
9. Tikhonov regularized optic flow reconstruction expressed in covariant derivatives. In order to formulate Tikhonov regularization in covariant derivatives, we first have to derive a gauge field. Such a gauge field imposes an a priori balance between velocity magnitude and velocity field changes and thereby it is supposed to be close to the velocity field that we would like to reconstruct from a sparse set of features. There are several options to choose the gauge field as an initial guess for the velocity field. We propose the standard Tikhonov regularization reconstruction with standard derivatives (i.e., constant gauge field) as a gauge field for a subsequent Tikhonov regularization reconstruction using covariant derivatives. The latter step is then to be considered as a refinement of the first.

Next we explain the whole reconstruction procedure in formulas. Subsequently, we will motivate the use of covariant derivatives and we will solve and analyze the corresponding Euler-Lagrange equations.

Definition 9.1. Let $\Omega$ be an open, convex subset of $\mathbb{R}^{2}$ with a piecewise smooth boundary. Let $k \in\{1, \ldots, K\}$ denote a fixed time frame and let $\lambda>0$. Let $\left\{w_{q}^{k}\right\}_{q=1}^{N_{B}} \in$ $\left(\mathbb{R}^{+}\right)^{N_{B}}$ denote a given weight vector. Let $\mathbf{h}^{k}$ be a smooth gauge field on $\Omega$ and let the discrete (sparse velocity) vector

$$
\mathbf{d}^{k}:=\left(\mathbf{d}^{k, 1}, \mathbf{d}^{k, 2}\right)^{T} \in \mathbb{R}^{2 N_{B}}
$$

be given by $\mathbf{d}^{k, j}:=\left(d_{1}^{k, j}, d_{2}^{k, j}, \ldots, d_{N_{B}}^{k, j}\right)^{T} \in \mathbb{R}^{N_{B}}$, with components given by (3.6). Let

$$
\phi_{k}^{q}(\mathbf{x}):=\phi_{s_{q}\left(t_{k}\right)}\left(\mathbf{x}-\mathbf{x}_{s=s_{q}\left(t_{k}\right)}^{q}\right)
$$

denote the Gaussian kernel, (3.1), centered around $\mathbf{x}_{s=s_{q}\left(t_{k}\right)}^{q} \in \mathbb{R}^{2}$ with scale $s_{q}\left(t_{k}\right)>0$, recall Figure 5, such that

$$
\left(\phi_{k}^{q}, v^{k, j}\right)_{\mathbb{L}_{2}(\Omega)}=\left(\phi_{s=s_{q}\left(t_{k}\right)} * v^{k j}\right)\left(\mathbf{x}_{s=s_{q}\left(t_{k}\right)}^{q}\right)=d_{q}^{k, j},
$$

where $q \in\left\{1, \ldots, N_{B}\right\}$ enumerates the extremal branches and where $j \in\{1,2\}$ enumerates the vertical and horizontal components of the field.

Then we define the energy 3 of a smooth velocity field $\mathbf{v}^{k}$ on $\Omega$ by

$$
\begin{aligned}
\mathcal{E}^{\lambda, \mathbf{h}^{k}, \mathbf{d}^{k}}\left(\mathbf{v}^{k}\right) & :=\lambda \mathcal{E}_{r e g}^{\mathbf{h}^{k}}\left(\mathbf{v}^{k}\right)+\mathcal{E}_{\text {data }}^{\mathbf{d}^{k}}\left(\mathbf{v}^{k}\right) \\
& :=\lambda \int_{\Omega} \|\left.\left|D^{\mathbf{h}^{k}} \mathbf{v}^{k}(\mathbf{x})\right|\right|^{2} \mathrm{~d} \mathbf{x}+\sum_{q=1}^{N_{B}} w_{q}^{k} \sum_{j=1}^{2}\left|\left(\phi_{k}^{q}, v^{k, j}\right)_{\mathbb{L}_{2}(\Omega)}-d_{q}^{k, j}\right|^{2},
\end{aligned}
$$

where $\left\|\left|D^{\mathbf{h}^{k}} \mathbf{v}^{k}(\mathbf{x})\right|\right\|$ denotes the Hilbert-Schmidt norm of the tensor field $D^{\mathbf{h}^{k}} \mathbf{v}^{k}(\mathbf{x})=$ $\sum_{i, j=1}^{2} \partial_{x^{i}}^{h^{k, j}} v^{k, j}(\mathbf{x}) \mathrm{d} x^{i} \otimes \partial_{j}$, i.e.:

$$
\left\|\left|D^{\mathbf{h}^{k}} \mathbf{v}^{k}(\mathbf{x})\right|\right\|^{2}=\sum_{i=1}^{2} \sum_{j=1}^{2}\left|\partial_{x^{i}}^{h^{k, j}} v^{k, j}(\mathbf{x})\right|^{2} .
$$

The reconstruction procedure is as follows. The velocity field $\mathbf{v}^{k}=\mathbf{v}^{k, 1} \mathbf{e}_{1}+\mathbf{v}^{k, 2} \mathbf{e}_{2}$, at time step $k$, is obtained by first minimizing $\mathcal{E}^{\lambda, \mathbf{h}^{k}=\mathbf{1}, \mathbf{d}^{k}}\left(\mathbf{v}^{k}\right)$ when $\mathbf{h}^{k}=\mathbf{1}$ is constant, yielding an optimal field, say $\left(\mathbf{v}^{k}\right)^{*}$, which produces the gauge field $\mathbf{h}^{k}:=\left(\mathbf{v}^{k}\right)^{*}$ for

\footnotetext{
${ }^{3}$ Later in Section 13 we propose a minor adaptation for a fully rotation covariant energy.
} 
the second/final refinement step where $\mathcal{E}^{\lambda,\left(\mathbf{v}^{k}\right)^{*}, \mathbf{d}^{k}}\left(\mathbf{v}^{k}\right)$ is minimized. Within each minimization, the parameter $\lambda>0$ balances between the regularization part and the soft constraints weighted by $w_{q}^{k}>0$. In our algorithm we have set $w_{q}^{k}=1$.

The minimization takes place over the space $\mathbf{H}_{0}^{1}(\Omega)=\mathbb{H}_{0}^{1}(\Omega) \times \mathbb{H}_{0}^{1}(\Omega)$, so we shall assume that $\mathbf{v}$ vanishes at the boundary. In practical applications, where the field does not vanish at the boundary, we subtract the harmonic infilling $\tilde{\mathbf{v}}=\mathbf{v}-\left(\left.\mathbf{v}\right|_{\partial \Omega}\right)_{\mathcal{H}}$. In the remainder of this article we simply write $\mathbf{v} \in \mathbf{H}_{0}^{1}(\Omega)$ instead of $\tilde{\mathbf{v}} \in \mathbf{H}_{0}^{1}(\Omega)$.

For $\lambda>0$ and hard constraints $\left(w_{q}^{k} \rightarrow \infty\right)$ we arrive at the minimization framework on a space of Sobolev type, [11, ch:3.4.2.1], where we must set $\mathcal{R}=\left(\left(D^{\mathbf{h}^{k}}\right)^{*} D^{\mathbf{h}^{k}}+I\right)^{\frac{1}{2}}$ to construct the complete Sobolev space $\mathcal{D}(\mathcal{R})$ on which the minimization problem takes place. The unique minimizer is then found by orthogonal projection. Variation of $\lambda>0$ can considerably improve the reconstruction, likewise in Figure 4 (right column).

9.1. The advantage of covariant derivatives. By using covariant derivatives (w.r.t. the gauge field $\left.\left(\mathbf{v}^{k}\right)^{*}\right)$, instead of regular derivatives, we employ the same velocity features twice: both in the data term and in the regularization term. The sparser the set of features, the more dominant the regularization term becomes. When using covariant derivatives w.r.t. the gauge field $\left(\mathbf{v}^{k}\right)^{*}$, the regularization term prefers (fields close to) the gauge field (which is based on the features) over constant fields which are in general not based on the features.

9.2. The Euler-Lagrange equations for the unique minimizer. Recall from Section 8 that the covariant Laplacian $\left(D^{\mathbf{h}^{k}}\right)^{*} D^{\mathbf{h}^{k}}: \mathbf{H}_{0}^{2}(\Omega) \rightarrow \mathbf{L}_{2}(\Omega)$ is coercive. Thereby the energy minimization problem (9.4) over the Hilbert space $\mathbf{H}_{0}^{2}(\Omega)$ (compactly and densely embedded in $\mathbf{L}_{2}(\Omega)$ ) consists (after diagonalization, Theorem 8.1) of a convex lower semicontinuous functional so that the existence and uniqueness of the minimizer is guaranteed by the general results in [15, Thm. 1, pp. 524-526].

The Euler-Lagrange equation for the unique minimizer of (9.4) is derived by

$$
\lim _{\epsilon \rightarrow 0} \frac{\mathcal{E}^{\lambda, \mathbf{h}^{k}, \mathbf{d}^{k}}\left(\mathbf{v}^{k}+\epsilon \boldsymbol{\delta}\right)-\mathcal{E}^{\lambda, \mathbf{h}^{k}, \mathbf{d}^{k}}\left(\mathbf{v}^{k}\right)}{\epsilon}=0,
$$

which is supposed to hold for all infinitely smooth perturbations that are compactly supported within the interior of $\Omega$, i.e. $\delta \in \mathcal{D}(\Omega)$.

Lemma 9.2. The Euler-Lagrange equation in the general continuous Tikhonov regularization framework using covariant derivatives (i.e. the minimization of (9.4)) is given by

$$
\left(\lambda\left(D^{\mathbf{h}^{k}}\right)^{*} D^{\mathbf{h}^{k}}+\mathcal{S}_{k}^{*} \Lambda_{k} \mathcal{S}_{k}\right) \mathbf{v}^{k}=\mathcal{S}_{k}^{*} \Lambda_{k} \mathbf{d}^{k},
$$

where $\mathcal{S}_{k}: \mathbf{L}_{2}(\Omega) \rightarrow \mathbb{R}^{2 \times N_{B}}$ is given by

$$
\left(\mathcal{S}_{k} \mathbf{v}^{k}\right)(q)=\left(\phi_{k}^{q}, \mathbf{v}^{k}\right):=\left(\phi_{k}^{q}, v^{k, 1}\right)_{\mathbb{L}_{2}(\Omega)} \mathbf{e}_{1}+\left(\phi_{k}^{q}, v^{k, 2}\right)_{\mathbb{L}_{2}(\Omega)} \mathbf{e}_{2},
$$

and where $\Lambda_{k} \in \mathbb{R}^{N_{B} \times N_{B}}$ is the diagonal matrix consisting of the corresponding weights:

$$
\Lambda_{k}=\underset{q=1, \ldots, N_{B}}{\operatorname{diag}}\left\{\left(w_{q}^{k}\right)\right\}
$$

Proof. A straightforward computation of (9.5) yields

$$
{ }^{\forall} \boldsymbol{\delta} \in \mathcal{D}(\Omega) 2\left(\left(\lambda\left(D^{\mathbf{h}^{k}}\right)^{*} D^{\mathbf{h}^{k}}\right) \mathbf{v}^{k}+\mathcal{S}_{k}^{*} \Lambda_{k} \mathcal{S}_{k} \mathbf{v}^{k}-\mathcal{S}_{k}^{*} \Lambda_{k} \mathbf{d}^{k}, \boldsymbol{\delta}\right)=0,
$$


from which the result follows.

The adjoint $\mathcal{S}_{k}^{*}: \mathbb{R}^{2 \times N_{B}} \rightarrow \mathbf{L}_{2}(\Omega)$ operator for each fixed discrete time $k \in \mathbb{N}$ is defined by

$$
\left(\mathcal{S}_{k}^{*} \Phi, \mathbf{v}^{k}\right)_{\mathbf{L}_{2}(\Omega)}=\left(\Phi, \mathcal{S}_{k} \mathbf{v}^{k}\right)_{\mathbb{R}^{2 \times N_{B}}}
$$

and by straightforward manipulation (see [12, ch:8, p.24]) we find

$$
\left(S_{k}^{*} \Phi\right)(\mathbf{x})=\sum_{j=1}^{2} \mathbf{e}_{j}\left(\sum_{q=1}^{N_{B}}(\Phi(q))^{j} \phi_{k}^{q}(\mathbf{x})\right) .
$$

This allows us to write down the Euler-Lagrange equations (9.6) in a more explicit form:

$$
\begin{aligned}
& \sum_{q=1}^{N_{B}} w_{q}^{k}\left(\left(\phi_{k}^{q}, v^{k, j}\right)_{\mathbb{L}_{2}(\Omega)}-d_{q}^{k, j}\right) \phi_{k}^{q}(\mathbf{x}) \\
& \quad+\lambda\left(-\Delta\left|v^{k, j}\right|(\mathbf{x})+\eta \frac{\Delta\left|h^{k, j}\right|(\mathbf{x})}{\left|h^{k, j}\right|(\mathbf{x})} v^{k, j}(\mathbf{x})\right)=0
\end{aligned}
$$

for $j=1,2, \mathbf{x} \in \mathbb{R}^{2}$, with $\eta=1$. Recall from Section 6.3 that we can interpolate between regular and covariant derivatives by varying the parameter $\eta \in[0,1]$. We will use (9.10) as a starting point for our implementations where all field components are expanded in a B-spline basis. For stability analysis we prefer the more structured form (9.6) where the whole operator is bounded from below (by the results in Sections 7 \& 8) so that

$$
\left(\left(\lambda\left(D^{\mathbf{h}^{k}}\right)^{*} D^{\mathbf{h}^{k}}+\mathcal{S}_{k}^{*} \Lambda_{k} \mathcal{S}_{k}\right) \mathbf{v}, \mathbf{v}\right)_{\mathbf{L}_{2}(\Omega)}>\mathbf{c}(\Omega) \lambda(\mathbf{v}, \mathbf{v})_{\mathbf{L}_{2}(\Omega)}
$$

and thereby operator $\lambda\left(D^{\mathbf{h}^{k}}\right)^{*} D^{\mathbf{h}^{k}}+\mathcal{S}_{k}^{*} \Lambda_{k} \mathcal{S}_{k}$ is invertible. Summarizing, we have proved the following result:

Theorem 9.3. The unique minimizer of the minimization problem (9.4) is given by

$$
\mathbf{v}^{k}=\left(\lambda\left(D^{\mathbf{h}^{k}}\right)^{*} D^{\mathbf{h}^{k}}+\mathcal{S}_{k}^{*} \Lambda_{k} \mathcal{S}_{k}\right)^{-1} \mathcal{S}_{k}^{*} \Lambda_{k} \mathbf{d}^{k}
$$

where $\mathcal{S}_{k}: \mathbf{L}_{2}(\Omega) \rightarrow \mathbb{R}^{2 \times N_{B}}$ is given by (9.7), $\mathcal{S}_{k}^{*}$ is given by (9.9), $\Lambda_{k}$ is given by (9.8) and $\mathbf{d}^{k}$ is given by (9.1) and (9.3).

10. Reconstruction algorithm: Solving the Euler-Lagrange equations by expansion in B-splines. Next we express the Euler-Lagrange equations entirely in B-spline coefficients. The computational advantages of using B-splines for variational approaches are well known in signal image processing, [21, 31, 57]. We will first provide a few basic properties on B-splines that we will need for our algorithm and the analysis of its stability later on.

The $n$ th-order (centered) B-spline is given by $(n-1)$-fold convolution of $B^{0}$ with itself:

$$
B^{n}(x)=\left(B^{0} *^{n-1} B^{0}\right)(x) \text { with } B^{0}(x)=1_{\left[-\frac{1}{2}, \frac{1}{2}\right]}(x),
$$

where $(f * g)(x)=\int_{-\infty}^{\infty} f(y) g(x-y) \mathrm{d} y$. Thereby the $n$ th-order B-spline is compactly supported on $1_{\left[-\frac{n}{2}-\frac{1}{2}, \frac{n}{2}+\frac{1}{2}\right]}$. In the discrete setting we sample a uniform integer grid, so for example if $n$ is odd we find $n$ nonzero samples. The regular derivative of a B-spline of order $n$ is expressed in B-splines of order $n-1$,

$$
\frac{d}{d x} B^{n}(x)=B^{n-1}(x+1 / 2)-B^{n-1}(x-1 / 2) .
$$


Consequently, for even-order derivatives of B-splines we have

$$
\left(B^{n}\right)^{(2 k)}(x)=\sum_{l=-k}^{k}(-1)^{l}\left(\begin{array}{c}
k \\
|l|
\end{array}\right) B^{n-2 k}(x-l) .
$$

Next we express the unknown velocities $\tilde{v}^{k, j}: \Omega \rightarrow \mathbb{R}, j=1,2$, at time frame $t=k \Delta t$, in periodic B-splines:

$$
\begin{aligned}
& \tilde{v}^{k, j}(x, y)=\sum_{l=0}^{L-1} \sum_{m=0}^{M-1} c_{l m}^{k, j} b^{m l}(x, y), \text { with } \\
& b^{m l}(x, y)=B^{n}\left(\frac{x}{a}-m \operatorname{Mod} \frac{M}{a}\right) B^{n}\left(\frac{y}{b}-l \bmod \frac{L}{b}\right),
\end{aligned}
$$

for all $(x, y) \in \Omega=[0, M] \times[0, L]$, where the field $\tilde{\mathbf{v}}^{k}=\mathbf{v}^{k}-\left(\left.\mathbf{v}^{k}\right|_{\partial \Omega}\right)_{\mathcal{H}}$ is indeed periodic.

In our algorithms we set the resolution parameters $a=b=1$. One can choose them differently, as in [31, provided that the $n$ th-order B-splines are properly sampled on $[0, M] \times[0, L]$, i.e.

$$
\frac{M}{a}>n+1 \text { and } \frac{L}{b}>n+1 .
$$

Definition 10.1. We define the discrete energy corresponding to the continuous en$\operatorname{ergy} \mathcal{E}_{\lambda, \mathbf{h}^{k}, \mathbf{d}^{k}}\left(\mathbf{v}^{k}\right)$ given in Definition 9.1 by

$$
E_{\lambda, \mathbf{h}^{k}, \mathbf{d}^{k}}\left(\mathbf{c}^{k}, \mathbf{d}^{k}\right):=\mathcal{E}_{\lambda, \mathbf{h}^{k}, \mathbf{d}^{k}}\left(\mathbf{v}^{k}\right),
$$

where $\mathbf{v}^{k}$ is expanded in the B-spline basis (10.2) and where

$$
\begin{gathered}
\mathbf{c}^{k}=\left(\left(\mathbf{c}^{k, 1}\right)^{T},\left(\mathbf{c}^{k, 2}\right)^{T}\right)^{T}=\left(c_{11}^{k, 1}, c_{12}^{k, 1}, \ldots, c_{1 M}^{k, 1}, c_{21}^{k, 1}, c_{22}^{k, 1}, \ldots, c_{2 M}^{k, 1}, \ldots, \ldots, c_{L 1}^{k, 1}, c_{L 2}^{k, 1}, \ldots, c_{L M}^{k, 1} ;\right. \\
\left.c_{11}^{k, 2}, c_{12}^{k, 2}, \ldots, c_{1 M}^{k, 2}, c_{21}^{k, 2}, c_{22}^{k, 2}, \ldots, c_{2 M}^{k, 2}, \ldots, \ldots, c_{L 1}^{k, 2}, c_{L 2}^{k, 2}, \ldots, c_{L M}^{k, 2}\right)^{T} \in \mathbb{R}^{2 M L} .
\end{gathered}
$$

Lemma 10.2. Let $k \in\{1, \ldots, K\}$ be a given time frame index. The discrete energy at time $k \Delta t$ can be expressed directly in the B-spline coefficients:

$$
E_{\lambda, \mathbf{h}^{k}, \mathbf{d}^{k}}\left(\mathbf{c}^{k}, \mathbf{d}^{k}\right):=\sum_{j=1}^{2}\left(\mathbf{c}^{k j}, R_{k, j}^{\lambda} \mathbf{c}^{k j}\right)_{\ell_{2}(\{1, \ldots, L M\})}+\left\|\Lambda_{k}^{1 / 2}\left(S \mathbf{c}^{k, j}-\mathbf{d}^{k, j}\right)\right\|_{\ell_{2}\left(\left\{1, \ldots, N_{B}\right\}\right)}^{2},
$$

where the $\mathbb{R}^{M L \times M L}$ matrices $R_{k, j}^{\lambda}$, for $j=1,2$, are given by

$$
\begin{aligned}
R_{k, j}^{\lambda}= & \lambda\left(-T_{L, 0}\left(\frac{y}{b}\right) \otimes T_{M, 2}\left(\frac{x}{a}\right)-T_{L, 2}\left(\frac{y}{b}\right) \otimes T_{M, 0}\left(\frac{x}{a}\right)\right. \\
& +\lambda \sum_{x=0}^{M-1} \sum_{y=0}^{L-1} \gamma_{k, j}(x, y)\left(\tilde{T}_{L, 0}\left(\frac{y}{b}\right) \otimes \tilde{T}_{M, 0}\left(\frac{x}{a}\right)\right)
\end{aligned}
$$

where $\left(\mathbf{c}^{k, j}, R_{L, 0} \otimes R_{M, 0} \mathbf{c}^{k, j}\right)=\sum_{l, l^{\prime}=0}^{L-1} \sum_{m, m^{\prime}}^{M-1} c_{l m}^{k, j} c_{l^{\prime} m^{\prime}}^{k, j}\left(R_{L, 0}\right)_{l l^{\prime}}\left(R_{M, 0}\right)_{m m^{\prime}}$ and $A \otimes B$ denotes the Kronecker product of matrices and where

$$
\begin{aligned}
\tilde{T}_{P, k}(u) & =\left[\tilde{T}_{P, k}^{p p^{\prime}}(u)\right] \in \mathbb{R}^{P \times P} & \text { with } \tilde{T}_{P, k}^{p p^{\prime}}(u):=\left(B^{n}\right)^{(k)}(u-p) B^{n}\left(u-p^{\prime}\right), \\
T_{P, k} & =\left[T_{P, k}^{p p^{\prime}}\right] \in \mathbb{R}^{P \times P} & \text { with } T_{P, k}^{p p^{\prime}}:=\left(B^{2 n}\right)^{(k)}\left(p-p^{\prime} \bmod P\right),
\end{aligned}
$$


with $p, p^{\prime} \in\{1, \ldots, P\}, P \in\{L, M\}$. The functions $\gamma_{k, j}: \Omega \rightarrow \mathbb{R}$ are given by

$$
\gamma_{k, j}(x, y)=\frac{\Delta h^{k, j}(x, y)}{h^{k, j}(x, y)}=-\Delta\left(-\log \left|h^{k, j}\right|\right)(x, y)+\left\|\nabla \log \left|h^{k, j}\right|(x, y)\right\|^{2} .
$$

Proof. By property (10.1) and assuming (10.3) we have the following formula for the components $T_{P K}^{p p^{\prime}}$ of the rank-2 tensor $T_{P, K}$ on $\mathbb{R}^{P}$ :

$$
\begin{aligned}
T_{P, k}^{p p^{\prime}} & :=\frac{1}{a} \int_{0}^{P}\left(B^{n}\right)^{(k)}\left(\frac{x}{a}-p \bmod \frac{P}{a}\right) B^{n}\left(\frac{x}{a}-p^{\prime} \bmod \frac{P}{a}\right) \mathrm{d} x \\
& =\frac{1}{a} \int_{-\frac{P}{2}}^{\frac{P}{2}}\left(B^{n}\right)^{(k)}\left(\frac{x}{a}-p \bmod \frac{P}{a}\right) B^{n}\left(\frac{x}{a}-p^{\prime} \bmod \frac{P}{a}\right) \mathrm{d} x \\
& =\left(B^{2 n}\right)^{(k)}\left(p-p^{\prime} \bmod P\right),
\end{aligned}
$$

with $P \in\{M, L\}$. The matrix representation of the covariant Laplacian expressed in the B-spline basis is given by (10.7), where

$$
\left(\mathbf{c}^{k, j}, R_{L, 0} \otimes R_{M, 0} \mathbf{c}^{k, j}\right)=\sum_{l, l^{\prime}=0}^{L-1} \sum_{m, m^{\prime}}^{M-1} c_{l m}^{k, j} c_{l^{\prime} m^{\prime}}^{k, j}\left(R_{L, 0}\right)_{l l^{\prime}}\left(R_{M, 0}\right)_{m m^{\prime}}
$$

and $A \otimes B$ denotes the Kronecker product of matrices. The rest follows by direct computation [12].

Next we derive the discrete analogue of Lemma 9.2. To this end we express the mapping $\mathcal{S}$ in (9.7) in B-spline coefficients $\mathbf{c}^{k}$ :

$$
\mathcal{S}_{k} \mathbf{c}^{k}(q)=\sum_{j=1}^{2} \mathbf{e}_{j}\left(\sum_{l=0}^{L-1} \sum_{m=0}^{M-1} c_{l m}^{k, j}\left(\phi_{k}^{q}, b^{m l}\right)\right) .
$$

If we expand the feature vectors $\left\{\phi_{k}^{q}\right\}_{q=1}^{N_{B}}$ as well as $\phi_{k}^{q}=\sum_{m^{\prime}=1}^{M-1} \sum_{l^{\prime}=0}^{L-1} \tilde{c}_{l^{\prime} m^{\prime}}^{k, q} b^{m^{\prime} l^{\prime}}$, then we may rewrite the mapping $\mathcal{S}_{k}: \mathbb{R}^{2 L M} \rightarrow \mathbb{R}^{q}$ as

$$
\mathcal{S}_{k} \mathbf{c}^{k}(q)=\sum_{l, l^{\prime}=0}^{L-1} \sum_{m, m^{\prime}=0}^{M-1} \tilde{c}_{l m^{\prime}}^{k q} T_{M, 0}^{m m^{\prime}} T_{L, 0}^{l l^{\prime}} c_{l m}^{k j}=\left(\tilde{\mathbf{c}}^{k q}\right)^{T}\left(T_{L, 0} \otimes T_{M, 0}\right) \mathbf{c}^{k j} .
$$

Theorem 10.3. Let the order $n$ of the B-spline basis be $n \leq 3$. Then the unique minimizer of the discrete functional (10.6) is given by

$$
\mathbf{c}^{k, j}=\left(R_{k, j}^{\lambda}+S_{k}^{T} \Lambda_{k} S_{k}\right)^{-1} S_{k}^{T} \Lambda_{k} \mathbf{d}^{k, j},
$$

using the natural matrix representation $S_{k} \in \mathbb{R}^{N_{B} \times L M}$ of the isomorphic mappings $\mathbf{c}^{k 1} \mapsto \mathcal{S}_{k}\left(\mathbf{c}^{k 1}, \mathbf{0}\right)$ and $\mathbf{c}^{k 2} \mapsto \mathcal{S}_{k}\left(\mathbf{0}, \mathbf{c}^{k 2}\right)$.

Proof. To compute the unique minimizer we set $\nabla_{\mathbf{c}^{k}} E_{\lambda, h^{k}, d^{k}}\left(\mathbf{c}^{k}\right)=\mathbf{0}$, yielding

$$
\left(R_{k, j}^{\lambda}+S_{k}^{T} \Lambda_{k} S_{k}\right) \mathbf{c}^{k, j}=S_{k}^{T} \Lambda_{k} \mathbf{d}^{k, j} \text { for } j=1,2,
$$

with $\mathbf{d}^{k, j}$ given by (9.1) and (9.3). The discrete system (10.10) minimizing the discrete functional (10.6) is consistent with (9.11) minimizing the continuous functional (9.4). Its solution coincides with the unique solution of the continuous system if the velocity components are constrained to the (closed) linear span

$$
\operatorname{span}_{m=0, \ldots, M-1, l=0, \ldots, L-1}\left\{b^{m l}\right\} .
$$


For $n \leq 3$, it will follow by the results in the next section (Corollary 11.2) that the system is well-posed and its unique solution is (akin to (9.11) ) found by the inversion in (10.9) and the result follows.

REMARK 10.4. We solved (10.9) by a BiGCSTAB algorithm, [51, ch:7.4.2]. Here we have exploited the direct product structure of the terms in the matrix $R_{k}^{\lambda}$ (10.7): For numerical efficiency one only needs to store the product $M \times M$ or $L \times L$ matrices such as $T_{M, 0}$ and $T_{L, 0}$ using the computation scheme explained in [27] in the BiGCSTAB algorithm whenever a matrix product occurs.

11. Stability analysis of the linear system in the Euler-Lagrange equations. In this subsection we will analyze the stability of the inversion scheme (10.9) which is basically the matrix representation of the operator equation (9.11) where we restrict ourselves to the subspace (10.11). By Theorem 9.3 the continuous operator

$$
\lambda\left(D^{\mathbf{h}^{k}}\right)^{*} D^{\mathbf{h}^{k}}+\mathcal{S}_{k}^{*} \Lambda_{k} \mathcal{S}_{k}
$$

can be inverted in (9.11) and we have

$$
\lambda\left(D^{\mathbf{h}^{k}}\right)^{*} D^{\mathbf{h}^{k}} \geq \lambda c_{\mathbf{h}^{k}} \text { and } \mathcal{S}_{k}^{*} \Lambda_{k} \mathcal{S}_{k} \geq 0,
$$

where $c_{\mathbf{h}^{k}}$ is the Poincaré constant of the covariant Laplacian, which depends on the choice of gauge field $\mathbf{h}^{k}$. In practice (where gauge fields are convex in large subsets of $\Omega$ ) this Poincaré constant is larger than the Poincaré constant of the regular Laplacian; recall Example 7.3

Let us return to the finite matrix representations $R_{k, j}^{\lambda}+S^{T} \Lambda_{k} S, j=1,2$, of operator (11.1) with respect to the periodic B-spline basis (10.2), where we recall (10.7). We would like to get estimates for the smallest eigenvalue of this finite matrix and we would like to investigate how this smallest eigenvalue depends on the order of the B-splines. First of all we observe that the data matrix $S^{T} \Lambda_{k} S \in \mathbb{R}^{N_{B} \times L M}$ is only positive semi-definite with $L M \gg N_{B}$, so even in the case where all features are linearly independent we should not expect global stability from the data matrix.

Lemma 11.1. Let the order $n$ of the B-splines be less than or equal to 3 . Let $\lambda>0$, $k \in\{1, \ldots, K\}, j \in\{1,2\}$. The real value of the eigenvalues of matrix $R_{k, j}^{\lambda}$ is larger than

$$
c_{\mathbf{h}^{k}} \lambda\left(\frac{6863}{23040}\right)^{2}>0,
$$

where $c_{\mathbf{h}^{k}}>0$ denotes the Poincaré constant of the covariant Laplacian on $\Omega$, which is usually larger than the Poincaré constant of the regular Laplacian on $\Omega$ (e.g. $\pi^{2}\left(M^{-2}+\right.$ $L^{-2}$ ) on a rectangle $\left.\Omega=[0, M] \times[0, L]\right)$.

Proof. The regularization matrix $R_{k, j}^{\lambda}$ for each component $j \in\{0,1\}$ satisfies

$$
R_{k, j}^{\lambda} \geq c_{\mathbf{h}^{k}} \lambda T_{L, 0} \otimes T_{M, 0}
$$


with $T_{L, 0}$ and $T_{M, 0}$ given by (10.8), since substitution of (10.2) into $(\mathbf{v}, \mathbf{v})_{\mathbb{L}_{2}(\Omega)}, \Omega=$ $[0, M] \times[0, L]$, gives

$$
\begin{aligned}
& \sum_{j=1}^{2}\left(\mathbf{c}^{k j}, R_{k, j}^{\lambda} \mathbf{c}^{k j}\right)_{\ell_{2}(\{1, \ldots, L M\})}=\left(\lambda\left(D^{\mathbf{h}^{k}}\right)^{*} D^{\mathbf{h}^{k}} \mathbf{v}, \mathbf{v}\right)_{\mathbb{L}_{2}(\Omega)} \\
& \geq c_{\mathbf{h}^{k}} \lambda(\mathbf{v}, \mathbf{v})_{\mathbb{L}_{2}(\Omega)}=c_{\mathbf{h}^{k}} \lambda \sum_{j=1}^{2}\left(\mathbf{c}^{k j}, T_{L, 0} \otimes T_{M, 0} \mathbf{c}^{k j}\right)_{\ell_{2}(\{1, \ldots, L M\})} .
\end{aligned}
$$

Now (11.2) relates the stability of matrix $T_{L, 0} \otimes T_{M, 0}$ with inverse $\left(T_{L, 0} \otimes T_{M, 0}\right)^{-1}=$ $T_{L, 0}^{-1} \otimes T_{M, 0}^{-1}$ to the stability of $R^{\lambda_{k, j}}$. The eigenvalues of $T_{L, 0} \otimes T_{M, 0}$ are direct products of the eigenvalues of $T_{L, 0}$ with the eigenvalues of $T_{M, 0}$, so in order to find a lower bound of the smallest eigenvalue of $R_{k, j}^{\lambda}$ we derive the smallest eigenvalue of $T_{P, 0}$ with $P \in$ $\{M, L\}$. Now $T_{P, 0}=\left[T_{P, 0}\right]^{p p^{\prime}}=\left[B^{2 n}\left(p-p^{\prime} \bmod P\right)\right]^{p p^{\prime}}$ is a circulant Toeplitz matrix whose columns add up to one. This matrix is not symmetric (due to periodicity), so its eigenvalues need not be real-valued. It is positive definite though and since

$$
\left(\mathbf{c}^{k j}, T_{L, 0} \otimes T_{M, 0} \mathbf{c}^{k j}\right)_{\ell_{2}(\{1, \ldots, L M\})}=\left(\mathbf{c}^{k j}, \frac{1}{2}\left(T_{L, 0} \otimes T_{M, 0}+\left(T_{L, 0} \otimes T_{M, 0}\right)^{T}\right) \mathbf{c}^{k j}\right)_{\ell_{2}(\{1, \ldots, L M\})},
$$

we only need to consider the real part of the eigenvalues $\operatorname{Re}(\lambda)=\frac{1}{2}(\lambda+\bar{\lambda})$. The Gerschgorin circle theorem [24] shows that the eigenvalues are contained within the circles

$$
\left|\lambda-B^{2 n}(0)\right| \leq 1-B^{2 n}(0) \Leftrightarrow \sqrt{\left|\operatorname{Re}(\lambda)-B^{2 n}(0)\right|^{2}+|\operatorname{Im}(\lambda)|^{2}} \leq 1-B^{2 n}(0),
$$

where we note that $2 B^{2 n}(0)-1$ is strictly positive iff $n \leq 3$. The eigenvalues of circulant Toeplitz matrices can be computed explicitly (eigenvalues of a circulant Toeplitz matrix coincide with the Discrete Fourier Transform (DFT) of the first row):

$$
\begin{aligned}
\lambda_{p} & =\sum_{p^{\prime}=0}^{P-1} B^{2 n}\left(p^{\prime}\right) e^{\frac{-2 \pi i p^{\prime} p}{P}}, \\
\operatorname{Re}\left(\lambda_{p}\right) & =B^{2 n}(0)+\frac{1}{2}\left(Z B^{2 n}\right)\left(e^{\frac{-2 \pi i p}{P}}\right),
\end{aligned}
$$

with corresponding eigenvector $\left(1, e^{-\frac{2 \pi i p}{P}}, \ldots, e^{-\frac{2 \pi i p(P-1)}{P}}\right)^{T} \in \mathbb{C}^{P}, p=0, \ldots, P-1$. The Z-transform of $B^{2 n}$ is given by $\left(Z B^{2 n}\right)(z)=\sum_{p^{\prime}=-n}^{n} c_{p^{\prime}}^{2 n} z^{-p^{\prime}}$, with $c_{p^{\prime}}^{2 n} \in \mathbb{R}^{+}$monotonically decreasing with $c_{0}^{2 n}=B^{2 n}(0)$ and as a result we can estimate the eigenvalues

$$
B^{2 n}(0)+\sum_{p^{\prime}=1}^{P-1}(-1)^{p^{\prime}} c_{p^{\prime}}^{2 n}=\operatorname{Re}\left(\lambda_{p=\frac{P}{2}}\right) \leq \operatorname{Re}\left(\lambda_{p}\right) \leq \operatorname{Re}\left(\lambda_{0}\right)=1 .
$$

The lower bound depends on $n$. Exact computations for $n \leq 10$ indicate that it is monotonically decreasing with $n$. We found $\frac{5}{8}, \frac{155}{384}, \frac{6863}{23040} \approx 0.298$, for $n=1,2,3$.

Corollary 11.2. The system in Theorem 10.3 is well-posed.

In our experiments we have set $n=3, M=L=93$ and $\lambda=10^{0.06} \approx 1.15$ and we had $c_{\mathbf{h}^{k}}>\pi^{2}\left(M^{-2}+L^{-2}\right) \approx 0.0023$. 
12. Algorithm. The overall algorithm combines

(1) the sparse vector field obtained by scale selection of critical point velocities as described in Section 3 ,

(2) the multi-scale Helmholtz decomposition considered in Section 4, Theorem 4.5,

(3) the Euler-Lagrange equations of Tikhonov regularization expressed in covariant derivatives as considered, analytically and numerically solved in respectively Section 9, Theorem 9.3 and Section 10.

Recall Figure 2. We first compute the scale space representation $(x, y, s, t) \mapsto I(x, y, s, t)$, and the critical branches $\left\{s \mapsto\left(\mathbf{x}_{s}^{q}(t), s\right)\right\}_{q=1}^{N_{B}}$ therein, of the preprocessed tagged MRIimages. For the critical branches we use a method based on winding numbers [53] for sub-pixel accuracy [12. Then we determine the points $\left(\mathbf{x}_{s=s_{q}(t)}, s^{q}(t)\right)$ on these branches by means of the scale selection criterium (3.3). At these points we compute a sparse set of velocities $\mathbf{d}_{q}^{k}$ and associated feature vectors $\phi_{k}^{q}$ according to (3.5), (3.6), and (9.2).

This sparse set of velocities is distributed over the divergence-free and rotation-free parts as follows. We apply a reconstruction by means of a Tikhonov regularization scheme with standard derivatives and very small $0<\lambda \ll 1$ to obtain a (full) regularized velocity field $\mathbf{v}^{k}$ that (nearly) satisfies the hard constraints. Then we apply multi-scale Helmholtz decomposition using the analytic vector-valued kernels from Theorem 4.5

$$
\begin{aligned}
\mathbf{d}^{k} & =\left(\left(\phi_{s_{q}\left(t_{k}\right)} * \mathbf{v}^{k}\right)\left(\mathbf{x}_{s=s_{q}(t)}\right)\right)_{q=1 \ldots N_{B}} \\
& =\left(\left(\phi_{s_{q}\left(t_{k}\right)} * \mathbf{v}^{k, \mathrm{rf}}\right)\left(\mathbf{x}_{s=s_{q}\left(t_{k}\right)}\right)\right)_{q=1 \ldots N_{B}}+\left(\left(\phi_{s_{q}\left(t_{k}\right)} * \mathbf{v}^{k, \mathrm{df}}\right)\left(\mathbf{x}_{s=s_{q}\left(t_{k}\right)}\right)\right)_{q=1 \ldots N_{B}} \\
& =\left(\sum_{j=1}^{2}\left(\mathbf{k}_{j}^{\mathrm{rf}, s_{q}\left(t_{k}\right)} * v^{k, j}\right)\left(\mathbf{x}_{s=s_{q}\left(t_{k}\right)}\right)\right)_{q=1 \ldots N_{B}}+\left(\sum_{j=1}^{2}\left(\mathbf{k}_{j}^{\mathrm{df}, s_{q}\left(t_{k}\right)} * v^{k, j}\right)\left(\mathbf{x}_{s=s_{q}\left(t_{k}\right)}\right)\right)_{q=1 \ldots N_{B}} \\
& =: \mathbf{d}^{k, \mathrm{rf}}+\mathbf{d}^{k, \mathrm{df}}
\end{aligned}
$$

to split the sparse feature vector $\mathbf{d}^{k}$ (cf. (9.1), (9.3) and (3.1)), at each time frame $t_{k}=k \Delta t$. This decomposition corresponds to the rightmost green box in Figure 2 , Furthermore, the red boxes in Figure 2 correspond to minimizing the energy (9.4) in Definition 9.1 by means of (9.11) in Theorem 9.3 . More precisely, the red boxes correspond to minimization of the discrete energy (10.6) in Definition 10.1 obtained by expansion in a $B$-spline basis, solved by means of (10.9) in Theorem 10.3. The final result is obtained by adding the reconstruction from the divergence-free gauge fields $\mathbf{h}^{k, \mathrm{df}}$ and $\mathbf{d}^{k, \mathrm{df}}$ to the reconstruction from the rotation-free gauge fields $\mathbf{h}^{k, \mathrm{rf}}$ and $\mathbf{d}^{k, \mathrm{rf}}$.

13. Rotation covariance. Our algorithm commutes with translations; i.e., translation of input image $f: \mathbb{R}^{2} \times \mathbb{R}^{+} \rightarrow \mathbb{R}$ results in a translated optical flow vector field $\mathbf{v}$. With respect to rotations this commutation property is not a priori satisfied. Subsequently, we show how to adapt the energy to obtain a fully rotation-covariant algorithm.

Rotation of the input scalar field is given by $f \mapsto f_{R}$ whereas rotation of the output optic flow vector field is given by $\mathbf{v}^{k} \mapsto \mathbf{v}_{R}^{k}, k=1, \ldots, K$, where

$$
f_{R}(\mathbf{x}, t)=f\left(R^{-1} \mathbf{x}, t\right) \text { and } \mathbf{v}_{R}(\mathbf{x}, t)=R \mathbf{v}\left(R^{-1} \mathbf{x}, t\right) .
$$


Our algorithm is rotation-covariant if

$$
\mathcal{E}^{\lambda, \mathbf{h}_{R}^{k},\left(R \mathbf{d}_{q}^{k}\right)_{q=1}^{N_{B}}}\left(\mathbf{v}_{R}^{k}\right)=\mathcal{E}^{\lambda, \mathbf{h}^{k},\left(\mathbf{d}_{q}^{k}\right)_{q=1}^{N_{B}}\left(\mathbf{v}^{k}\right)}
$$

for all $R \in S O(2), k=1, \ldots, K$. If $R$ is a rotation over $n \pi / 2$, with $n \in \mathbb{Z}$, then (13.1) is satisfied; see [12, ch.8.2]. For the other cases it depends on the spherical fluctuations between the fraction of slope and height of the graph of the projected (gauge) field, where projections take place on each orientation $\mathbf{n} \in S^{2}$.

Strict rotation covariance is obtained by replacing $\mathcal{E}_{r e g}^{\mathbf{h}^{k}}\left(\mathbf{v}^{k}\right)$, cf. (9.4), with

$$
\tilde{\mathcal{E}}_{r e g}^{\mathbf{h}^{k}}\left(\mathbf{v}^{k}\right)=\int_{0}^{2 \pi}\left\|D^{\mathbf{h}^{k}(\mathbf{x}) \cdot \mathbf{n}(\theta)}\left(\mathbf{v}^{k}(\mathbf{x}) \cdot \mathbf{n}(\theta)\right)\right\|^{2} \mathrm{~d} \theta
$$

with $\mathbf{n}(\theta)=(\cos \theta, \sin \theta)^{T}$. In the algorithm this boils down to replacing

$$
\begin{gathered}
\left(D^{\mathbf{h}^{k}}\right)^{*} D^{\mathbf{h}^{k}} \mathbf{v}^{k}(\mathbf{x})=\sum_{i, j=1}^{2}\left(\left(\frac{\partial}{\partial x^{i}}\right)^{h^{k, j}}\right)^{*}\left(\frac{\partial}{\partial x^{i}}\right)^{h^{k, j}} v^{k, j}(\mathbf{x}) \mathbf{e}_{j} \longrightarrow \\
\int_{0}^{2 \pi}\left(\left(D^{h_{\theta}^{k}}\right)^{*} D^{h_{\theta}^{k}} v_{\theta}^{k}\right)(\mathbf{x}) \mathbf{n}(\theta) \mathrm{d} \theta=\int_{0}^{2 \pi}\left(-\Delta v_{\theta}^{k}(\mathbf{x})+\frac{\Delta h_{\theta}^{k}(\mathbf{x})}{h_{\theta}^{k}(\mathbf{x})} v_{\theta}^{k}(\mathbf{x})\right) \mathbf{n}(\theta) \mathrm{d} \theta
\end{gathered}
$$

where $v_{\theta}^{k}:=\mathbf{v}^{k} \cdot \mathbf{n}(\theta)$ and $h_{\theta}^{k}:=\mathbf{h}^{k} \cdot \mathbf{n}(\theta)$.

Note that $\mathcal{E}_{r e g}^{\mathbf{h}^{k}}\left(\mathbf{v}^{k}\right)$ is a course sampling of $\tilde{\mathcal{E}}_{r e g}^{\mathbf{h}^{k}}\left(\mathbf{v}^{k}\right)$ (sampled at $\left.\left\{0, \frac{\pi}{2}, \pi, \frac{3 \pi}{2}\right\}\right)$. Recall Remark 6.1 For properly defined 2-tensors $t$ on a circle, one has $\int_{0}^{2 \pi} t(\mathbf{n}(\theta), \mathbf{n}(\theta)) \mathrm{d} \theta=$ $\sum_{k \in\{0,1\}} t\left(\mathbf{n}\left(k \frac{\pi}{2}\right), \mathbf{n}\left(k \frac{\pi}{2}\right)\right)=$ trace $(t)$. However, a series of experiments in [12, Fig.12] show that (13.2) has a minor correcting effect and the replacement (13.3) is not needed.

14. A single Euler-Lagrange system involving covariant derivatives and Helmholtz decomposition. In this section we will consider a single Euler-Lagrange system involving both multi-scale Helmholtz decomposition and covariant derivatives. We will see a direct analogy with our approach explained in Section 12 and Figure 2. The approach in this section has the advantage that it splits the divergence-free and rotationfree parts more consistently, although it is much more cumbersome to implement.

In the sequel we shall denote the divergence-free part of the optical flow vector field $\mathbf{v}^{k}$ at the $k$ th time frame by $\mathbf{v}^{k, \text { df }}$ and the rotation-free part by $\mathbf{v}^{k, \mathrm{rf}}$.

At a given fixed time frame $t=k \Delta t$ we minimize the following positive functional:

$$
\begin{gathered}
\mathcal{E}^{\lambda, \mathbf{h}^{k}, \mathbf{d}^{k}}\left(\mathbf{v}^{k}\right):=\left.\int_{\Omega} \lambda\left\||| D^{\left(\mathbf{h}^{k}\right)^{\mathrm{df}}} \mathbf{v}^{k, \mathrm{df}}(\mathbf{x})\right\|\right|^{2} \mathrm{~d} \mathbf{x}+\int_{\Omega} \lambda \| \mid D^{\left(\mathbf{h}^{k}\right)^{\mathrm{rf}} \mathbf{v}^{k, \mathrm{rf}}(\mathbf{x}) \|\left.\right|^{2} \mathrm{~d} \mathbf{x}} \\
+\sum_{q=1}^{N_{B}} w_{q}^{k} \sum_{j=1}^{2}\left|\left(\phi_{k}^{q}, v^{k, j}\right)_{\mathbb{L}_{2}(\Omega)}-d_{q}^{k, j}\right|^{2},
\end{gathered}
$$

where ||$|\cdot|||$ denotes the Hilbert-Schmidt norm, and where we recall the previous definitions in Section 9 We again first consider the simple case $\mathbf{h}_{k}=(1,1)^{T}$ (where all covariant derivatives become standard derivatives) to construct our gauge field $\mathbf{h}^{k}$.

Theorem 14.1. The unique minimizer $\mathbf{v}=\mathbf{v}_{k}$ at the $k$ th time frame of the problem

$$
\min _{\left.\substack{\mathbf{v} \in \mathbf{H}_{0}^{1}(\Omega), \\ \text { s.t. } \quad \mathbf{v} f}\right|_{\partial \Omega}=\left.\mathbf{v}^{\mathrm{rf}}\right|_{\partial \Omega}=\mathbf{0}} \mathcal{E}^{\lambda, \mathbf{h}^{k}, \mathbf{d}^{k}}(\mathbf{v}),
$$


with positive, convex functional given by (14.1), is given by $\mathbf{v}^{k}=\nabla \phi+\widetilde{\operatorname{rot}} A$, where $\phi \in \mathbb{H}_{0}^{2}(\Omega)$ is the unique weak solution of the PDE-system:

$$
\begin{aligned}
& -\lambda \Delta^{2} \phi+\operatorname{div}\left(\left(\lambda \underset{j=1,2}{\operatorname{diag}}\left\{\frac{\Delta h^{\mathrm{rf}, j}}{h^{\mathrm{rf}, j}}\right\}+\mathcal{S}_{k}^{*} \Lambda_{k} \mathcal{S}_{k}\right)\left(\begin{array}{c}
\frac{\partial \phi}{\partial x} \\
\frac{\partial \phi}{\partial y}
\end{array}\right)\right)+\operatorname{div}\left(\mathcal{S}_{k}^{*} \Lambda_{k} \mathbf{d}^{\mathrm{rf}}\right)=0, \\
& \left.\phi\right|_{\partial \Omega}=0 \text { and }\left.\frac{\partial \phi}{\partial n}\right|_{\partial \Omega}=0
\end{aligned}
$$

and where $A \in \mathbb{H}_{0}^{2}(\Omega)$ is the unique weak solution of the PDE-system:

$$
\begin{aligned}
& \lambda \Delta^{2} A+\operatorname{rot}\left(\left(\underset{j=1,2}{\left.\lambda \underset{j i a g}{\operatorname{din}^{\mathrm{df}, j}}\right\}}\left\{+\mathcal{S}_{k}^{*} \Lambda_{k} \mathcal{S}_{k}\right)\left(\begin{array}{c}
\frac{\partial A}{\partial y} \\
-\frac{\partial A}{\partial x}
\end{array}\right)\right)+\operatorname{rot}\left(\mathcal{S}_{k}^{*} \Lambda_{k} \mathbf{d}^{\mathrm{df}}\right)=0,\right. \\
& \left.A\right|_{\partial \Omega}=0 \text { and }\left.\frac{\partial A}{\partial n}\right|_{\partial \Omega}=0 .
\end{aligned}
$$

Proof. Due to convexity, strict positivity and lower semi-continuity of the functional, the minimizer is unique [15, Thm.1, pp.524-526]. Computing the first-order variation of the energy given by (14.1) with respect to an arbitrary smooth perturbation $\delta \in(\mathcal{D}(\Omega))^{2}$,

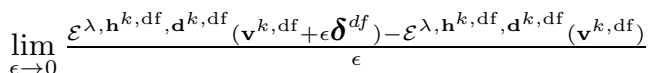

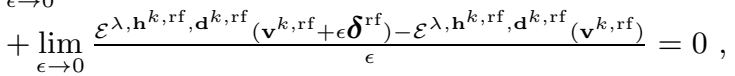

yields the following EL-equations (akin to Theorem 9.3):

$$
\left\{\begin{array}{l}
\left.\lambda\left(D^{\mathbf{h}^{k, \mathrm{rf}}}\right)^{*} D^{\mathbf{h}^{k, \mathrm{rf}}} \mathbf{v}^{k, \mathrm{rf}}+\mathcal{S}_{k}^{*} \Lambda_{k} \mathcal{S}_{k}\left(\mathbf{v}^{k, \mathrm{rf}}\right)+\mathcal{S}_{k}^{*} \Lambda_{k} \mathbf{d}^{k, \mathrm{rf}}, \boldsymbol{\delta}^{\mathrm{rf}}\right)=0, \\
\left.\lambda\left(D^{\mathbf{h}^{k, \mathrm{df}}}\right)^{*} D^{\mathbf{h}^{k, \mathrm{df}}} \mathbf{v}^{k, \mathrm{df}}+\mathcal{S}_{k}^{*} \Lambda_{k} \mathcal{S}_{k}\left(\mathbf{v}^{k, \mathrm{df}}\right)+\mathcal{S}_{k}^{*} \Lambda_{k} \mathbf{d}^{k, \mathrm{df}}, \boldsymbol{\delta}^{\mathrm{df}}\right)=0 .
\end{array}\right.
$$

As rotation-free vector fields are $\mathbf{L}_{2}$-orthogonal to divergence-free vector fields we find

$$
\begin{aligned}
& \operatorname{div}\left(\lambda\left(D^{\mathbf{h}^{k, \mathrm{rf}}}\right)^{*} D^{\mathbf{h}^{k, \mathrm{rf}}} \mathbf{v}^{k, \mathrm{rf}}+\mathcal{S}_{k}^{*} \Lambda_{k} \mathcal{S}_{k}\left(\mathbf{v}^{k, \mathrm{rf}}\right)+\mathcal{S}_{k}^{*} \Lambda_{k} \mathbf{d}^{k, \mathrm{rf}}\right)=0, \\
& \operatorname{rot}\left(\lambda\left(D^{\mathbf{h}^{k, \mathrm{df}}}\right)^{*} D^{\mathbf{h}^{k, \mathrm{df}}} \mathbf{v}^{k, \mathrm{df}}+\mathcal{S}_{k}^{*} \Lambda_{k} \mathcal{S}_{k}\left(\mathbf{v}^{k, \mathrm{df}}\right)+\mathcal{S}_{k}^{*} \Lambda_{k} \mathbf{d}^{k, \mathrm{df}}\right)=0
\end{aligned}
$$

under the constraints

$$
\operatorname{rot}\left(\mathbf{v}^{k, \mathrm{rf}}\right)=\mathbf{0} \text { and } \operatorname{div}\left(\mathbf{v}^{k, \mathrm{df}}\right)=0,
$$

from which (14.2) and (14.3) follow, since div $\circ \nabla=\Delta$ and rot $\widetilde{\operatorname{rot}}=-\Delta$ and recall (6.9). By means of the Green's identities it can be shown, akin to [15, p.345], that the PDE-systems have unique weak solutions in $\mathbb{H}_{0}^{2}(\Omega)$; e.g., one has

$$
\begin{aligned}
& -\lambda \int_{\Omega} \Delta \phi(\mathbf{x}) \Delta \psi(\mathbf{x}) \mathrm{d} \mathbf{x} \\
& +\int_{\Omega} \psi(\mathbf{x})\left(\operatorname{div}\left(\left(\lambda \underset{j=1,2}{\lambda \operatorname{diag}}\left\{\frac{\Delta h^{\mathrm{rf}, j}}{h^{\mathrm{rf}, j}}\right\}+\mathcal{S}_{k}^{*} \Lambda_{k} \mathcal{S}_{k}\right)\left(\begin{array}{c}
\frac{\partial \phi}{\partial x} \\
\frac{\partial \phi}{\partial y}
\end{array}\right)\right)+\operatorname{div}\left(\mathcal{S}_{k}^{*} \Lambda_{k} \mathbf{d}^{\mathrm{rf}}\right)\right)(\mathbf{x}) \mathrm{d} \mathbf{x}=0
\end{aligned}
$$

for all $\psi \in \mathbb{H}_{0}^{2}(\Omega)$, from which the result follows.

If we now compare our approach in Section 12 and Figure 2, where we solved separate Euler-Lagrange equations for the diverence-free and rotation-free parts, we see that in that approach we did not have the divergence and rotation operator in (14.6) and we have dropped the constraints (14.7). Experiments in our technical report [12] show that the reconstruction from the divergence-free gauge field $\mathbf{h}^{k, \mathrm{df}}$ and divergence-free features $\mathbf{d}^{k, \mathrm{df}}$ in Figure 2 is not strictly divergence-free. The same holds for the reconstruction from the rotation-free gauge field $\mathbf{h}^{k, \mathrm{df}}$ and rotation-free features $\mathbf{d}^{k, \mathrm{df}}$. The approach in this section does not suffer from this problem. 
15. Experiments. In this section we will provide a single example of cardiac motion estimation of one healthy volunteer and of a patient whose heart is infarcted at several areas. Acquisition of heart images has been performed during the systolic phase (i.e. the phase of the heartbeat in which the heart muscle contracts to pump blood to the rest of the body); we assess 11 frames with a size of $86 \times 86$ pixels and pixel size of 1.37 $\mathrm{mm}^{2}$ with a slice-thickness of $8 \mathrm{~mm}$ and a temporal resolution of 0.02 seconds. In Figure 10 we respectively show the sampled motion field (column 1), rotation-free (column 2) and divergence-free (column 3) parts of frames 3, 6 and 8 for the healthy heart case (rows 1,2,3) and the diseased case (rows 4,5,6) respectively. By means of the Helmholtz decomposition we observe that the healthy cardiac muscle starts to systole with intense rotation (row 1, column 3) and little contraction (row 1, column 2). At mid-systole, the contribution of rotation-free parts becomes qualitatively similar to the contribution of the divergence-free part; that is, vectors inside the cardiac walls are of similar length (row 2, columns 2 and 3). At the end of the systolic phase, contraction becomes more relevant (row 3, column 2), whereas rotation is nearly absent (row 3, column 3). In the investigated diseased heart case, the heart exerts modest rotation through the whole sequence (rows 4,5,6 and column 3), leaving contraction as the dominant contribution to the heart cycle (rows 4,5,6 and column 2).

For further detailed experiments of our algorithm on analytic ground truth phantoms, comparisons between different related optic flow methods and comparisons between different parameter settings, we refer to our technical report [12] and [4]. These experiments show the practical benefits of both including multi-scale Helmholtz decompositions and covariant derivatives in our optical flow estimations.

In [4, Ch.5] our method is also compared to other established optic flow methods [44, 29, 59] by applying it to an artificial phantom dataset (cf. [4, Ch.5.2], also used in [58]) for which the ground truth is known. Our method performed best in terms of average angular errors [3] and magnitude errors; cf. [4, Table 5.1, Table 5.2].

Appendix A. Derivation of the Green's function of the 1D covariant Laplacian and related (in)equalities. In this section we derive the Green's function and prove equalities (8.3), (8.4), (8.5) and (8.6).

Let $h \in C^{2}([0,1]), h>0$ and consider the following PDE system on $[0,1]$ :

$$
\left(D^{h}\right)^{*} D^{h} f=\delta_{y} \text { and } f \in \mathbb{H}_{0}^{1}([0,1]) \text {, i.e. } f \in \mathbb{H}^{1}([0,1]) \text { and } f(0)=f(1)=0 \text {. }
$$

By the Lax-Milgram theorem, this problem has a unique solution; point evaluation $\psi \mapsto$ $\delta_{y}(\psi)=\psi(y)$ is a continuous linear functional on $\mathbb{H}_{0}^{1}([0,1])$ and the left-hand side is a bounded, coercive sesquilinear form on $\mathbb{H}_{0}^{1}([0,1])$. Now we set $\phi:=D^{h} f \in \mathbb{L}_{2}([0,1])$. Then $\left(D^{h}\right)^{*} \phi=0$ implies that $-\phi^{\prime}-\frac{h^{\prime}}{h} \phi=0$, i.e. $\phi h^{\prime}+\phi^{\prime} h=0 \Leftrightarrow h(x) \phi(x)=c_{1}$ for all $x \in[0,1]$ and some $c_{1} \in \mathbb{R}$. The substitution $\phi(x)=f^{\prime}(x)-\frac{h^{\prime}(x)}{h(x)} f(x)$ yields

$$
f^{\prime}(x) h(x)-h^{\prime}(x) f(x)=c_{1}
$$



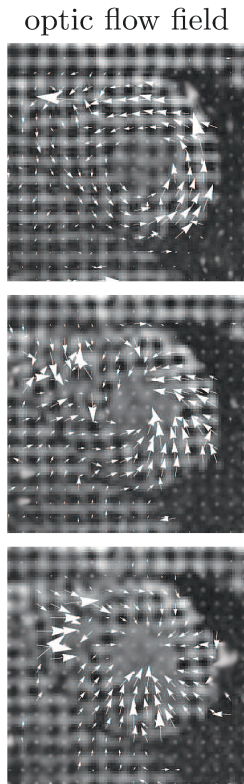

optic flow field
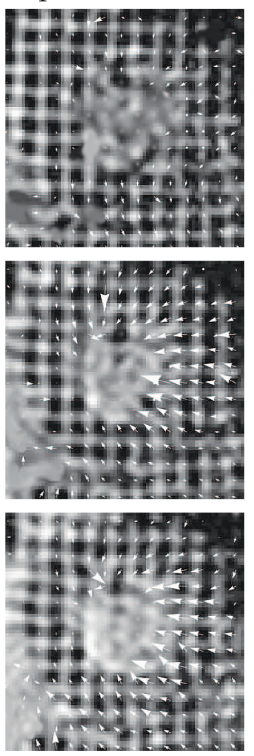

Healthy volunteer

rot-free part
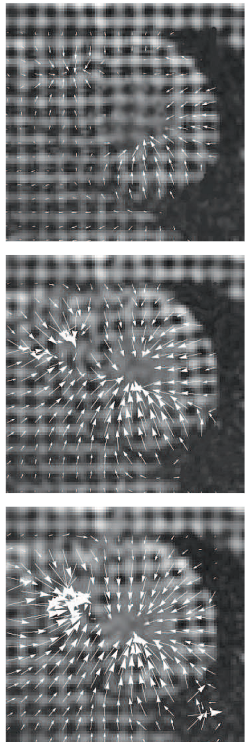

Patient

rot-free part
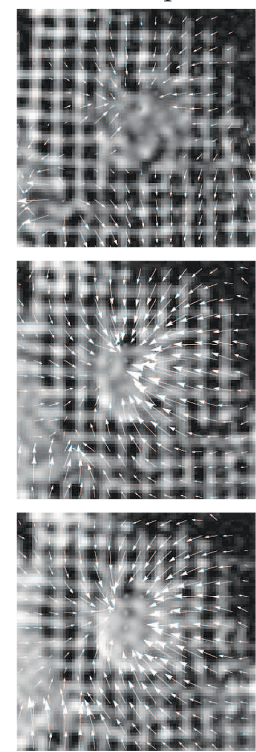

div-free part
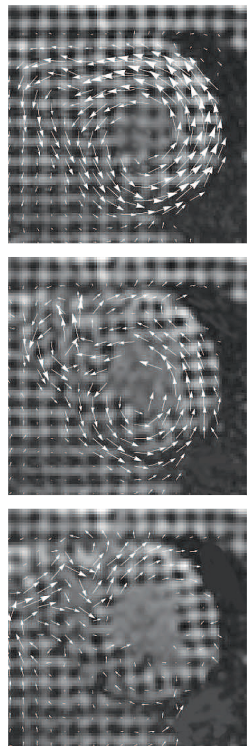

div-free part
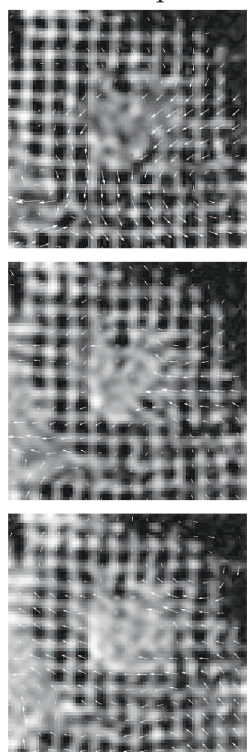

FIG. 10. Cardiac motion field behavior for a healthy volunteer and a patient. We assess time frames 3,6 and 8 (rows 1,2 and 3 healthy volunteer, rows 4,5 and 6 patient) of a sequence of 11 frames displaying the cardiac muscle during systole. Column 1 shows the extracted motion fields, column 2 shows the rotation-free part, whereas column 3 shows the divergence-free part. 
This first-order ODE has as homogeneous solution $f=c_{2} h$ and by the variation of constants formula we find the following standard solutions of the ODE $\left(D^{h}\right)^{*} D^{h} f=0$ :

$$
f(x)=c_{2} h(x)+c_{1} h(x) Q_{h}(x), \text { with } Q_{h}(x):=\int_{0}^{x} h(v)^{-2} \mathrm{~d} v .
$$

Now we find solutions $f_{0}$ and $f_{1}$ with $f_{0}(0)=0, f_{0}(1)=1, f_{1}(0)=1$ and $f_{1}(1)=0$ :

$$
f_{0}(x)=\frac{h(x) Q_{h}(x)}{h(1) Q_{h}(1)} \text { and } f_{1}(x)=\frac{h(x)\left(Q_{h}(1)-Q_{h}(x)\right)}{h(0) Q_{h}(1)} .
$$

The (constant) Wronskian of these solutions equals $W=f_{0}^{\prime}(0) f_{1}(0)-f_{0}(0) f_{1}^{\prime}(0)=$ $\frac{1}{Q_{h}(1) h(1) h(0)}$. Now the kernel operator $K_{h}$ given by (8.3) and (8.2) can be written as

$$
\begin{aligned}
& k(x, y)=\frac{1}{W}\left\{\begin{array}{ll}
f_{0}(x) f_{1}(y) & x \leq y, \\
f_{0}(y) f_{1}(x) & x>y,
\end{array} \Rightarrow\right. \\
& \left(K_{h} f\right)(x)=\frac{1}{W}\left(f_{1}(x) \int_{0}^{x} f_{0}(y) f(y) \mathrm{d} y+f_{0}(x) \int_{x}^{1} f_{1}(y) f(y) \mathrm{d} y\right) .
\end{aligned}
$$

Operator $K_{h}$ is the right inverse of the operator $\left(D^{h}\right)^{*} D^{h}$, where we note that $K_{h} f \in$ $\mathbb{H}_{0}^{2}([0,1])$ and $\left(D^{h}\right)^{*} D^{h} K_{h} f=f$, since

$$
K f^{\prime \prime}(x)=\frac{1}{W}\left(f_{1}^{\prime \prime}(x) \int_{0}^{x} f_{0}(y) f(y) \mathrm{d} y-f_{0}^{\prime \prime}(x) \int_{x}^{1} f_{1}(y) f(y) \mathrm{d} y-W f(x)\right),
$$

so indeed $-(K f)^{\prime \prime}+\frac{h^{\prime \prime}}{h} K_{h} f=f$. Regarding (8.6) we note that $k_{h}(x, y)=k_{h}(y, x)$ and

$$
\begin{aligned}
\left\|K_{h}\right\|^{2} & \leq\left\|\mid K_{h}\right\|\left\|^{2}=\operatorname{trace}\left\{K_{h}^{*} K_{h}\right\}=\right\| k_{h} \|_{\mathbb{L}_{2}([0,1] \times[0,1])}^{2} \\
& \leq \frac{2}{\left(Q_{h}(1)\right)^{2}} \int_{0}^{1} \int_{0}^{y} h^{2}(x) h^{2}(y) Q_{h}^{2}(x)\left(Q_{h}(1)-Q_{h}(y)\right)^{2} \mathrm{~d} x \mathrm{~d} y \\
& =\frac{2}{\left(Q_{h}(1)\right)^{2}} \int_{0}^{1} h^{2}(y)\left(Q_{h}(1)-Q_{h}(y)\right)^{2}\left(\int_{0}^{y} h^{2}(x) Q^{2}(x) \mathrm{d} x\right) \mathrm{d} y \\
& \leq 2\left(Q_{h}(1)\right)^{2}\|h\|_{\mathbb{L}_{2}([0,1])}^{4}=2\left(\int_{0}^{1} h^{-2}(y) \mathrm{d} y\right)^{2}\left(\int_{0}^{1} h^{2}(y) \mathrm{d} y\right)^{2},
\end{aligned}
$$

where we used that $0 \leq Q(x) \leq Q(1)$.

A.1. Proof of Lemma 8.3. This follows from the fact that both $f_{0}$ and $f_{1}$ and their Wronskian are positive (due to $0 \leq Q(x) \leq Q(1)$ ), so for example suppose $x \leq y$; then $\frac{\partial^{2}}{\partial x^{2}} k_{h}(x, y)=W^{-1} f_{0}^{\prime \prime}(x) f_{1}(y)=\frac{h^{\prime \prime}(x)}{h(x)}\left(W^{-1} f_{0}(x) f_{1}(x)\right)$.

Acknowledgements. The authors gratefully thank Dr. J.J.M. Westenberg of Leiden University Medical Center for providing the cardiac MR-dataset that we used as input in Section 15] of this article.

\section{REFERENCES}

[1] G.B. Arfken and H.J. Weber, Mathematical methods for physicists, Academic Press, San Diego, 1995. MR1423357 (98a:00001)

[2] L. Axel and L. Dougherty, MR imaging of motion with spatial modulation of magnetization, Radiology 171 (1989), no. 3, 841-845.

[3] J.L. Barron, D.J. Fleet, and S. Beauchemin, Performance of optical flow techniques, IJCV 12 (1994), no. 1, 43-77.

[4] A. Becciu, Feature based estimation of myocardial motion from tagged MR images, Ph.D. thesis, Eindhoven University of Technology, Dept. of Biomedical Engineering, 2010. 
[5] A. Becciu, B.J. Janssen, H.C. van Assen, L.M.J. Florack, V. Roode, and B.M. ter Haar Romeny, Extraction of cardiac motion using scale-space features points and gauged reconstruction, CAIP '09: Proceedings of the 13th International Conference on Computer Analysis of Images and Patterns (Berlin, Heidelberg), Springer-Verlag, 2009, pp. 598-605.

[6] A. Bruhn, J. Weickert, T. Kohlber, and C. Schnoerr, A multigrid platform for real-time motion computation with discontinuity-preserving variational methods, IJCV 70 (2006), no. 3, 257-277.

[7] T. Corpetti, E. Mémin, and P. Pérez, Dense estimation of fluid flows, IEEE PAMI 24 (2002), no. 3, 365-380.

[8] A. Cuzol, P. Hellier, and E. Mémin, A low dimensional fluid motion estimator, International Journal of Computer Vision 75 (2007), no. 3, 329-349.

[9] J. Damon, Local Morse theory for solutions to the heat equation and Gaussian blurring, Journal of Differential Equations 115 (1995), no. 2, 368-401. MR1310937(96a:58025)

[10] T. Delhaas, J. Kotte, and A. van der Toorn, Increase in Left Ventricular Torsion-to-Shortening Ratio in Children With Valvular Aorta Stenosis., Magnetic Resonance in Medicine 51 (2004), 135139.

[11] R. Duits, Perceptual organization in image analysis, Ph.D. thesis, Eindhoven University of Technology, Department of Biomedical Engineering, The Netherlands, 2005.

[12] R. Duits, A. Becciu, B.J. Janssen, H.C. van Assen, L.M.J. Florack, and B.M. ter Haar Romeny, Cardiac motion estimation using covariant derivatives and Helmholtz decomposition, CASA-report 31, Eindhoven University of Technology, Department of Mathematics and Computer Science, 2010, http://www.win.tue.nl/casa/research/casareports/2010.html.

[13] R. Duits, H. Fuehr, B.J. Janssen, and L.C.M. Bruurmijn, Left-invariant reassignment and diffusion on Gabor transforms, (2011), Submitted.

[14] R. Duits, B.J. Janssen, F.M.W. Kanters, and L.M.J. Florack, Linear image reconstruction from a sparse set of alpha scale space features by means of inner products of Sobolev type, Lecture Notes in Computer Science, Springer-Verlag 3753 (2005), 96-111.

[15] L.C. Evans, Partial differential equations, Graduate Studies in Mathematics, Vol.19, Amer. Math. Soc., Providence, 1998. MR1625845 (99e:35001)

[16] L.M.J. Florack, Image structure, Computational Imaging and Vision, Kluwer Academic Publishers, Dordrecht, The Netherlands, 1997.

[17] Scale space representations locally adapted to the geometry of base and target manifold, Mathematical Methods for Signal and Image Analysis and Representation (L.M.J. Florack, R. Duits, M.-C. Jongbloed, G. van Lieshout, and L. Davies, eds.), Springer-Verlag, 2011, pp. 175-189.

[18] L.M.J. Florack and A. Kuijper, The topological structure of scale-space images, Journal of Mathematical Imaging and Vision 12 (2000), no. 1, 65-79. MR1746979 (2000m:68171)

[19] L.M.J. Florack, W. Niessen, and M. Nielsen, The intrinsic structure of optic flow incorporating measurements of duality, International Journal of Computer Vision 27 (1998), no. 3, 263-286.

[20] L.M.J. Florack and H.C. van Assen, A New Methodology for Multiscale Myocardial Deformation and Strain Analysis Based on Tagging MRI, International Journal of Biomedical Imaging (2010), 1-8, Published online http://downloads.hindawi.com/journals/ijbi/2010/341242.pdf.

[21] P.E. Forssen, Low and medium level vision using channel representations, Ph.D. thesis, Linkoping University, Dept. EE, Linkoping, Sweden, March 2004.

[22] D. Gabor, Theory of communication, J. IEE 93 (1946), no. 26, 429-457.

[23] T. Georgiev, Relighting, retinex theory, and perceived gradients, Proceedings of Mirage 2005, INRIA Rocquencourt, 2005.

[24] S. Gerschgorin, Ueber die abgrenzung der eigenwerte einer matrix, Izv. Akad. Nauk. USSR Otd. Fiz.-Mat. Nauk. 7 (1931), 550-559.

[25] C.W. Groetsch, Elements of applicable functional analysis, Marcel Dekker Inc., New York, Basel, 1980. MR:569746 (83m:46103)

[26] S. Gupta, E.N. Gupta, and J.L. Prince, Stochastic models for div-curl optical flow methods, IEEE Signal Processing Letters 3 (1996), 32-35.

[27] P.C. Hansen, J.G. Nagy, and D.P. O'Leary, Deblurring images: Matrices, spectra, and filtering, first ed., SIAM, 2006. MR2271138(2008d:94007)

[28] C.O. Horgan and S. Nemat-Nasser, Bounds on eigenvalues of Sturm-Liouville problems with discontinuous coefficients, Journal of Applied Mathematics and Physics 30 (1979), 77-86. MR526211 (80b:34020)

[29] B.K.P. Horn and B.G. Schunck, Determining optical flow, AI 17 (1981), 185-203. 
[30] T. Iijima, Basic theory on normalization of a pattern (in case of typical one-dimensional pattern), Bulletin of Electrical Laboratory 26 (1962), 368-388.

[31] B.J. Janssen, Representation and manipulation of images based on linear functionals, Ph.D. thesis, Eindhoven University of Technology, Eindhoven, The Netherlands, 2009.

[32] B.J. Janssen and R. Duits, Linear image reconstruction by Sobolev norms on the bounded domain, International Journal of Computer Vision. 84 (2009), no. 2, 205-219.

[33] B.J. Janssen, R. Duits, and L.M.J. Florack, Coarse-to-fine image reconstruction based on weighted differential features and background gauge fields, Lecture Notes in Computer Science 5567 (2009), $377-388$.

[34] B.J. Janssen, L.M.J. Florack, R. Duits, and B.M. ter Haar Romeny, Optic flow from multi-scale dynamic anchor point attributes, Image Analysis and Recognition, Third International Conference, ICIAR 2006 (Berlin) (A. Campilho and M. Kamel, eds.), Lecture Notes in Computer Science, vol. 4141, Springer-Verlag, September 2006, pp. 767-779.

[35] B.J. Janssen, F.M.W. Kanters, R. Duits, L.M.J. Florack, and B.M. ter Haar Romeny, A linear image reconstruction framework based on Sobolev type inner products., International Journal of Computer Vision 70 (2006), no. 3, 231-240.

[36] F.M.W. Kanters, T. Denton, A. Shokoufandeh, L.M.J. Florack, and B.M. ter Haar Romeny, Combining different types of scale space interest points using canonical sets, Scale Space and Variational Methods (Berlin) (F. Sgallari, A. Murli, and N. Paragios, eds.), Lecture Notes in Computer Science, vol. 4485, Springer-Verlag, 2007, pp. 374-385.

[37] F.M.W. Kanters, L.M.J. Florack, R. Duits, B. Platel, and B.M. ter Haar Romeny, Scalespacevis: $\alpha$-scale spaces in practice, Pattern Recognition and Image Analysis 17 (2007), no. 1.

[38] F.M.W. Kanters, B. Platel, L.M.J. Florack and B.M. ter Haar Romeny, Image reconstruction from multiscale critical points, Scale Space Methods in Computer Vision, 4th International Conference, Scale Space 2003 (Isle of Skye, UK) (Lewis Griffin and Martin Lillholm, eds.), Springer, June 2003, pp. $464-478$.

[39] J.J. Koenderink, The structure of images, Biol. Cybern. 50 (1984), 363-370. MR758126

[40] T. Kohlberger, E. Mémin, and P. Pérez, Variational dense motion estimation using the Helmholtz decomposition, Proc. Scale Space Conference (Griffin. L.D. and M. Lillholm, eds.), Lecture Notes in Computer Science, vol. 2695, Springer-Verlag, 2003, pp. 432-448.

[41] M. Lillholm, M. Nielsen, and L.D. Griffin, Feature-based image analysis, International Journal of Computer Vision 52 (2003), no. 2/3, 73-95.

[42] T. Lindeberg, Scale-space for discrete signals, PAMI 12 (1990), no. 3, 234-245.

[43] _ Scale-space theory in computer vision, The Kluwer International Series in Engineering and Computer Science, Kluwer Academic Publishers, 1994.

[44] B. Lucas and T. Kanade, An iterative image registration technique with application to stereo vision, DARPA, Image Process, vol. 21, 1981, pp. 85-117.

[45] P.D. Milgram and A.N. Lax, Parabolic equations, Contributions to the theory of partial differential equations, Annals of Math. Studies 30 (1954), 167-190. MR0067317 (16:709b)

[46] I. Mirsky, J.M. Pfeffer, and M.A. Pfeffer, The contractile state as the major determinant in the evolution of left ventricular dysfunction in the spontaneously hypertensive rat. Circulation Research, 53 (1983), 767-778.

[47] M. Nielsen and M. Lillholm, What features tell about images?, Lecture Notes in Computer Science 2106 (2001), 39-50.

[48] T. Nir, A.M. Bruckstein, and R. Kimmel, Over-Parameterized variational optical flow, International Journal of Computer Vision 76 (2008), no. 2, 205-216.

[49] N.F. Osman, W.S. McVeigh, and J.L. Prince, Cardiac motion tracking using sine harmonic phase (harp) magnetic resonance imaging, Magnetic Resonance in Medicine 42 (1999), no. 6, 1048-1060.

[50] W. Rudin, Functional analysis, McGraw-Hill, New York, 1973. MR0365062 (51:1315)

[51] Y. Saad, Iterative methods for sparse linear systems (2nd edition), SIAM, 2003. MR1990645 (2004h:65002)

[52] F. Sheehan, D. Stewart, H. Dodge, S. Mitten, E. Bolson and G. Brown, Variability in the measurement of regional left ventricular wall motion, Circulation 68 (1983), 550-559.

[53] J. Staal, S. Kalitzin, B.M. ter Haar Romeny, and M. Viergever, Detection of critical structures in scale space, Lecture Notes in Computer Science, vol. 1682, 1999, pp. 105-116. 
[54] B.M. ter Haar Romeny, Front-end vision and multi-scale image analysis: Multiscale computer vision theory and applications, written in mathematica, Computational Imaging and Vision, Kluwer Academic Publishers, Dordrecht, The Netherlands, 2003.

[55] J.P. Thirion, Image matching as a diffusion process: an analogy with Maxwell's demons, Medical Image Analysis 2 (1998), no. 3, 243-260.

[56] Y. Tong, S. Lombeyda, A.N. Hivani, and M. Desbrun, Discrete multiscale vector field decomposition, ACM Transactions on Graphics (TOG) 22 (2003), 445-452.

[57] M. Unser, Splines: A perfect fit for signal and image processing, IEEE Signal Processing Magazine 16 (1999), no. 6, 22-38.

[58] H.C. van Assen, L.M.J. Florack, F.J.J. Simonis, and J.J.M. Westenberg, Cardiac strain and rotation analysis using multi-scale optical flow, MICCAI workshop on Computational Biomechanics for Medicine 5, Springer-Verlag, 2011, pp. 91-103.

[59] H.C. van Assen, L.M.J. Florack, A. Suinesiaputra, J.J.M. Westenberg, and B.M. ter Haar Romeny, Purely evidence based multiscale cardiac tracking using optic flow, Proc. MICCAI 2007 workshop on Computational Biomechanics for Medicine II (K. Miller, K. D. Paulsen, A. A. Young, and P. M. F. Nielsen, eds.), 2007, pp. 84-93.

[60] H. von Helmholtz, Ueber integrale der hydrodynamischen gleichungen, welche den wirbelbewegungen entsprechen, Crelles J. 55 (1858), no. 25.

[61] J. Weickert, S. Ishikawa, and A. Imiya, On the history of Gaussian scale-space axiomatics, Gaussian Scale-Space Theory, Computational Imaging and Vision Series, Kluwer Academic Publisher, 1997, pp. 45-59.

[62] E.A. Zerhouni, D.M. Parish, W.J. Rogers, A. Yang, and E.P. Sapiro, Human heart: Tagging with $M R$ imaging a method for noninvasive assessment of myocardial motion, Radiology 169 (1988), no. $1,59-63$.

[63] H. Zimmer, A. Bruhn, J. Weickert, L. Valgaerts, A. Salgado, B. Rosenhahn, and H.-P. Seidel, Complementary optic flow, Proceedings of Energy Minimization Methods in Computer Vision and Pattern Recognition (EMMCVPR), Lecture Notes in Computer Science, Berlin, Springer-Verlag, vol. 5681, 2009, pp. 207-220. 